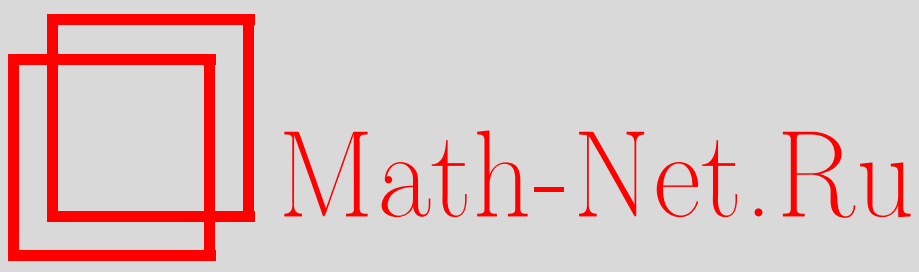

Л. М. Кожевникова, Стабилизация решения первой смешанной задачи для эволюционного квазиэллиптического уравнения, Матем. сб., 2005, том 196, номер 7, 67100

DOI: https://doi.org/10.4213/sm1377

Использование Общероссийского математического портала Math-Net.Ru подразумевает, что вы прочитали и согласны с пользовательским соглашением

http://www.mathnet.ru/rus/agreement

Параметры загрузки:

IP : 54.198 .64 .247

26 апреля 2023 г., 15:59:05 
УДК 517.956.4

\author{
Л. М. Кожевникова
}

\title{
Стабилизация решения первой смешанной задачи для эволюционного квазиэллиптического уравнения
}

В цилиндрической области $D=(0, \infty) \times \Omega$, где $\Omega \subset \mathbb{R}_{n+1}-$ неограниченная область, рассматривается первая смешанная задача для уравнения высокого порядка

$$
\begin{gathered}
u_{t}+L u=0, \\
L u \equiv \sum_{i=q}^{k}(-1)^{i} D_{x}^{i}\left(a_{i}(x, \mathbf{y}) D_{x}^{i} u\right)+\sum_{i=l}^{m} \sum_{|\alpha|=|\beta|=i}(-1)^{i} D_{\mathbf{y}}^{\alpha}\left(b_{\alpha \beta}(x, \mathbf{y}) D_{\mathbf{y}}^{\beta} u\right), \\
q \leqslant k, \quad l \leqslant m, \quad q, k, l, m \in \mathbb{N}, \quad x \in \mathbb{R}, \quad \mathbf{y} \in \mathbb{R}_{n},
\end{gathered}
$$

с однородньпи краевьми условиями и финитной начальной функцией. Предлагается новьй метод получения оценки сверху $L_{2}$-нормы $\|u(t)\|$ решения задачи, пригодньй для широкого класса областей и уравнений. В частности, для областей $\left\{(x, \mathbf{y}) \in \mathbb{R}_{n+1}:\left|y_{1}\right|<x^{a}\right\}, 0<a<q / l$, при некотором условии на символ оператора $L$ эта оценка принимает вид

$$
\|u(t)\| \leqslant M \exp \left(-\kappa_{2} t^{b}\right)\|\varphi\|, \quad b=\frac{q-l a}{q-l a+2 l a q} .
$$

Доказана точность оценки в шшроком классе неограниченных областей при $q=$ $k=l=m=1$, т.е. для параболического уравнения второго порядка.

Библиографория: 14 названий.

\section{Введение}

Пусть $\Omega$ - неограниченная область пространства $\mathbb{R}_{n+1}=\{(x, \mathbf{y}): x \in \mathbb{R}, \mathbf{y}=$ $\left.\left(y_{1}, y_{2}, \ldots, y_{n}\right) \in \mathbb{R}_{n}\right\}$. Ось $O x$ выделена в связи с конкретным видом рассматриваемого ниже уравнения. В цилиндрической области $D=\{t>0\} \times \Omega$ для уравнения

$$
\begin{gathered}
u_{t}+L u=0, \\
L u=\sum_{i=q}^{k}(-1)^{i} D_{x}^{i}\left(a_{i}(x, \mathbf{y}) D_{x}^{i} u\right)+\sum_{i=l}^{m} \sum_{|\alpha|=|\beta|=i}(-1)^{i} D_{\mathbf{y}}^{\alpha}\left(b_{\alpha \beta}(x, \mathbf{y}) D_{\mathbf{y}}^{\beta} u\right), \\
q \leqslant k, \quad l \leqslant m, \quad q, k, l, m \in \mathbb{N},
\end{gathered}
$$

Работа вьполнена при финансовой поддержке Российского фонда фундаментальных исследований (грант № 05-01-97912). 
рассмотрим первую смешанную задачу

$$
\begin{gathered}
\left.D_{x}^{i} u\right|_{S}=0, \quad i<k ;\left.\quad D_{\mathbf{y}}^{\alpha} u\right|_{S}=0, \quad|\alpha|<m ; \quad S=\{t>0\} \times \partial \Omega \\
u(0, x, \mathbf{y})=\varphi(x, \mathbf{y}), \quad \varphi \in L_{2}(\Omega)
\end{gathered}
$$

Здесь $\alpha, \beta$-мультииндексы, $\alpha=\left(\alpha_{1}, \alpha_{2}, \ldots, \alpha_{n}\right),|\alpha|=\alpha_{1}+\alpha_{2}+\cdots+\alpha_{n}$.

Переменную $t$ в уравнении будем называть временем. Функции $a_{i}(x, \mathbf{y}), i=$ $\overline{q, k}$, измеримы и для п.в. $(x, \mathbf{y}) \in \Omega$ ограничены сверху и снизу положительными константами

$$
\widehat{a} \leqslant a_{i}(x, \mathbf{y}) \leqslant \bar{a}, \quad i=\overline{q, k}, \quad 0<\widehat{a} \leqslant \bar{a} .
$$

Коэффициенты $b_{\alpha \beta}(x, \mathbf{y}),|\alpha|=|\beta|=\overline{l, m},-$ измеримые и для п.в. $(x, \mathbf{y}) \in \Omega$ ограниченные функции, симметричные по мультииндексам $\alpha, \beta$. Будем требовать при п.в. $x \in \mathbb{R}$ неотрицательность интеграла

$$
\sum_{i=l}^{m} \sum_{|\alpha|=|\beta|=i} \int_{\mathbb{R}_{n}} b_{\alpha \beta}(x, \mathbf{y}) D_{\mathbf{y}}^{\alpha} g D_{\mathbf{y}}^{\beta} g d \mathbf{y} \geqslant 0
$$

для любой функции $g(x, \mathbf{y})$ из $C_{0}^{\infty}(\Omega)$.

Отметим, что для коэффициентов $b_{\alpha \beta}(x)$, зависящих только от $x$, неравенство

$$
\sum_{i=l}^{m} \sum_{|\alpha|=|\beta|=i} b_{\alpha \beta}(x) \mathbf{z}^{\alpha} \mathbf{z}^{\beta} \geqslant 0
$$

справедливое для п.в. $x \in \mathbb{R}$ и всех $\mathbf{z} \in \mathbb{R}_{n}$, ввиду равенства Планшереля, обеспечивает вьполнение условия (0.5). Если коэффициенты $b_{\alpha \beta}(x, \mathbf{y})$ зависят и от $\mathbf{y}$, то для выполнения $(0.5)$ достаточно, чтобы

$$
\sum_{i=l}^{m} \sum_{|\alpha|=|\beta|=i} b_{\alpha \beta}(x, \mathbf{y}) z_{\alpha} z_{\beta} \geqslant 0
$$

для п.в. $(x, \mathbf{y}) \in \Omega$ и любого набора чисел $z_{\alpha}$.

Легко видеть, что среди уравнений вида (0.1) содержатся, кроме квазиэллиптических (см. определение в работе [1]), также и некоторые вырождающиеся уравнения.

Мы будем иметь дело с обобшенным решением задачи (0.1)-(0.3). В тех случаях, когда оно не обладает некоторыми из следов, входящих в краевые условия (0.2), связь обобщенного решения с классической постановкой задачи $(0.1)-(0.3)$ может теряться, но это не является препятствием для исследования поведения решения при $t \rightarrow \infty$.

Настоящая работа посвящена исследованию поведения при больших значениях времени $L_{2}(\Omega)$-нормы решения $u(t, x, \mathbf{y})$ задачи $(0.1)-(0.3)$. Предлагаются достаточно простые характеристики неограниченной области $\Omega$, в терминах которых получены оценки скорости убывания при $t \rightarrow \infty$ решения задачи $(0.1)-(0.3)$. 
Первые исследования зависимости скорости убывания решения смешанной задачи для параболического уравнения второго порядка от геометрии неограниченной области были выполнены А. К. Гушиньм в работах [2], [3]. Для широкого класса областей в них для решения второй смешанной задачи установлена оценка

$$
|u(t, \mathbf{y})| \leqslant \frac{\|\varphi\|_{L_{1}(Q)}}{v(\sqrt{t})}, \quad \mathbf{y} \in Q
$$

где $v(r)=\operatorname{mes}\{\mathbf{y} \in Q:|\mathbf{y}|<r\}$. Доказана также точность этой оценки. Аналогичный результат установлен В.И. Ушаковым в работе [4] для третьей смешанной задачи. Более полные исследования зависимости поведения при большом значении времени решения второй смешанной задачи от геометрии области и от начальной функции выполнены А.В. Лежневым в [5].

Исследованием поведения $L_{2}$-нормы решения смешанных задач для параболи-

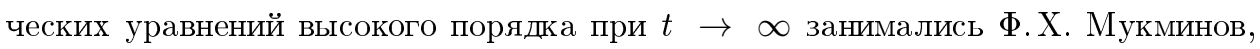
А.. Тедеев, И. М. Биккулов и др. В цитируемых ниже работах предполагалась финитность начальной функции $\operatorname{supp} \varphi \subset B\left(R_{0} ;(0,0)\right)$. (Здесь и далее через $B(r ;(x, \mathbf{y}))$ обозначаем шар радиуса $r$ с центром в точке $(x, \mathbf{y})$.) Отметим, что при отсутствии ограничений на носитель начальной функции скорость стабилизации решения зависит не только от области $\Omega$, но и от начальной функции $\varphi$ (см. [6]).

В работе [7] Ф.Х. Мукминовым рассматривалось уравнение

$$
u_{t}+(-1)^{m} \sum_{|\alpha|=|\beta|=m} D^{\alpha}\left(a_{\alpha \beta}(t, \mathbf{y}) D^{\beta} u\right)=0, \quad m \geqslant 1 .
$$

Коэффициенты уравнения - измеримые функции, удовлетворяюшие для п.в. $(t, \mathbf{y}) \in$ $(0, \infty) \times Q$ и любого набора чисел $z_{\alpha}$ неравенствам

$$
c_{1} \sum_{i=1}^{n} z_{m_{i}}^{2} \leqslant \sum_{|\alpha|=|\beta|=m} a_{\alpha \beta} z_{\alpha} z_{\beta} \leqslant c_{2} \sum_{|\alpha|=m} z_{\alpha}^{2},
$$

где $c_{1}, c_{2}$ - положительные постоянные, $m_{i}=(0, \ldots, m, \ldots, 0)$ - мультииндекс, $i$-я компонента которого равна $m$, остальные равны нулю. На область $Q$ налагались следующие требования: существует непрерывная монотонно невозрастающая функция $\mu(r), r>0$, удовлетворяющая условию

$$
\lim _{r \rightarrow \infty} r \mu^{1 /(2 m)}(r)=\infty
$$

и неравенству

$$
0<\mu(r) \leqslant \inf \left\{\int_{Q^{r}}\left|\sum_{i=1}^{n} D_{y_{i}}^{m} g\right|^{2} d \mathbf{y}: g(\mathbf{y}) \in C_{0}^{\infty}(Q), \quad \int_{Q^{r}} g^{2} d \mathbf{y}=1\right\},
$$

где $Q^{r}=\left\{\mathbf{y} \in Q:\left|y_{1}\right|<r\right\}$. При этих условиях для решения задачи $\left(0.1^{\prime}\right)$, $(0.2),(0.3)$ с финитной начальной функцией $\varphi$ при достаточно больших $t$ получена оценка

$$
\|u(t)\|_{L_{2}(Q)} \leqslant M \exp \left\{-k\left[\frac{r^{2 m}(t)}{t}\right]^{1 /(2 m-1)}\right\}\|\varphi\|_{L_{2}(Q)} .
$$


Здесь $r(t), t>0,-$ функция, обратная к функции $F(r)=r /[\mu(r)]^{(2 m-1) /(2 m)}$. Ранее в работе [8] $\Phi$. Х. Мукминовым была доказана аналогичная оценка для решения первой смешанной задачи в случае параболического уравнения второго порядка и доказана ее точность в классе неограниченных монотонно расширяюшихся областей врашения.

А.Ф. Тедеевьм [9] рассматривалось параболическое квазилинейное уравнение высокого порядка в дивергентной форме

$$
u_{t}+(-1)^{m} \sum_{|\alpha|=m} D^{\alpha} A_{\alpha}\left(t, \mathbf{y}, u, D u, \ldots, D^{m} u\right)=0, \quad m \geqslant 1
$$

Здесь $A_{\alpha}(t, \mathbf{y}, \xi)$ - каратеодориевы функции, удовлетворяющие условиям

$$
\begin{aligned}
& \sum_{|\alpha|=m} A_{\alpha}(t, \mathbf{y}, \xi) \xi_{\alpha}^{m} \geqslant c_{1} \sum_{|\alpha|=m}\left|\xi_{\alpha}^{m}\right|^{p}, \quad p \geqslant 2 \\
& \sum_{|\alpha|=m}\left|A_{\alpha}(t, \mathbf{y}, \xi)\right| \leqslant c_{2} \sum_{|\alpha|=m}\left|\xi_{\alpha}^{m}\right|^{p-1}
\end{aligned}
$$

для любого вектора $\xi=\left(\xi^{0}, \xi^{1}, \ldots, \xi^{m}\right), \xi^{i}=\left(\xi_{\alpha}^{i}\right),|\alpha|=i$, где $c_{1}, c_{2}$ - положительные постоянные.

Для области $Q$ предполагались выполненными следующие условия:

$$
\lim _{r \rightarrow \infty} \lambda(r)=0, \quad \lim _{r \rightarrow \infty} r^{2} \lambda(r)=\infty
$$

где $\lambda(r)$ - первое собственное значение задачи Дирихле в $Q^{r}$ для оператора $-\Delta$. При $p=2$ для решения задачи $\left(0.1^{\prime \prime}\right),(0.2),(0.3)$ с финитной начальной функцией $\varphi$ при достаточно больших $t$ получена оценка (0.6), в которой $r(t), t>0,-$ функция, обратная к функции $F(r)=r /[\lambda(r)]^{(2 m-1) / 2}$.

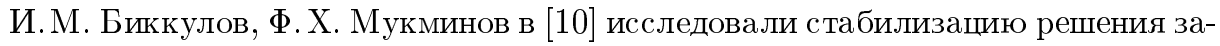
дачи Риккье для уравнений 4-го и 6-го порядков. В терминах функции $\lambda(r)$ ими получена оценка $L_{2}$-нормы решения при $t \rightarrow \infty$ и установлена ее точность по порядку стремления к нулю.

В работе [11] получены оценки решений систем квазилинейных параболических уравнений в терминах некоторых геометрических характеристик неограниченной области $\Omega$ и выделен класс областей, в которых эти оценки точны.

Интересно отметить, что в работе [12] получена оценка решения двумерной системы уравнений Навье-Стокса, аналогичная (0.6) с $m=1$.

Предлагаемьй нами метод получения оценок сверху для решения задачи (0.1)(0.3) позволяет единообразно рассматривать широкий класс эволюционных квазиэллиптических уравнений вида (0.1) в более широком классе неограниченных областей, чем в работах [8]-[12]. Точность полученных оценок в случае параболического уравнения второго поря дка устанавливается также для существенно более широкого класса областей, чем это было сделано в работах [8], [11].

Введем обозначение $\Omega_{a}^{b}=\{(x, \mathbf{y}) \in \Omega: a<x<b\}$, значения параметров $a=-\infty$ или $b=\infty$ могут быть опушены. В обозначении цилиндра $D_{c}^{d}=(c, d) \times \Omega$ значение $c=0$ может быть опушено. Положим $\gamma_{r}=\{(x, \mathbf{y}) \in \Omega: x=r\}$; 
предполагается, что $\gamma_{r} \neq \varnothing$ для всех $r>0$. Через $\|\cdot\|_{Q}$ обозначим норму в $L_{2}(Q)$, подразумевая, что $\|\cdot\| \equiv\|\cdot\|_{L_{2}(\Omega)}$.

Будем использовать также следующие обозначения:

$$
\begin{gathered}
F(g)=A(g)+B(g)=\sum_{i=q}^{k} a_{i}(x, \mathbf{y})\left(D_{x}^{i} g\right)^{2}+\sum_{i=l}^{m} \sum_{|\alpha|=|\beta|=i} b_{\alpha \beta}(x, \mathbf{y}) D_{\mathbf{y}}^{\alpha} g D_{\mathbf{y}}^{\beta} g, \\
\lambda\left(r_{1}, r_{2}\right)=\inf \left\{\int_{\Omega_{r_{1}}^{r_{2}}} F(g) d x d \mathbf{y}: g(x, \mathbf{y}) \in C_{0}^{\infty}(\Omega), \quad \int_{\Omega_{r_{1}}^{r_{2}}} g^{2} d x d \mathbf{y}=1\right\} \\
r_{1}<r_{2} .
\end{gathered}
$$

Для неотрицательных $\rho$ положим

$$
\rho^{[a, b]}=\left\{\begin{array}{ll}
\rho^{a}, & \rho<1, \\
\rho^{b}, & \rho \geqslant 1,
\end{array} \quad a, b>0 .\right.
$$

Неограниченную возрастаюшую последовательность положительных чисел $\left\{x_{j}\right\}_{j=0}^{\infty}$ назовем $\lambda$-последовательностью задачи (0.1)-(0.3), если существует число $\theta>0$ такое, что справедливы неравенства

$$
1 \leqslant \theta \lambda\left(x_{j}, x_{j+1}\right)\left(x_{j+1}-x_{j}\right)^{2[k, q]}, \quad j=\overline{0, \infty} .
$$

Приведем необходимое и достаточное условие сушествования $\lambda$-последовательности:

$$
\text { при любом } r_{1}>0 \text { найдется } r_{2}>r_{1} \text { такое, что } \lambda\left(r_{1}, r_{2}\right)>0
$$

(см. следствие 1). При этом $\lambda$-последовательность можно построить, начиная с любого $x_{0}>0$.

Ради некоторого упрошения формулировок результатов потребуем сушествование такого числа $x_{*}$, что $\lambda\left(-\infty, x_{*}\right)>0$. Нетрудно доказать, что это условие будет вьполнено и при любом $x>x_{*}$ (см. замечание после утверждения 2). Легко видеть, что при увеличении $R_{0}$ условие

$$
\operatorname{supp} \varphi \subset \Omega^{R_{0}}
$$

на носитель начальной функции остается справедливым. При необходимости можно также сдвигать нумерацию индексов в $\lambda$-последовательности, полагая $\widetilde{x}_{j}=x_{j+k}$. Поэтому, не ограничивая общность, в дальнейшем будем считать, что $R_{0}=x_{0}$ и

$$
\lambda\left(-\infty, x_{0}\right)>0
$$

Определим невозрастаюшую функцию дискретного аргумента

$$
\lambda(N)=\min \left\{\lambda\left(-\infty, x_{0}\right), \lambda\left(x_{0}, x_{1}\right), \ldots, \lambda\left(x_{N-1}, x_{N}\right)\right\}, \quad N \in \mathbb{N} .
$$

Тогда, очевидно, справедливо неравенство

$$
\lambda(N) \int_{\Omega^{x} N} g^{2} d x d \mathbf{y} \leqslant \int_{\Omega^{x} N} F(g) d x d \mathbf{y}, \quad g(x, \mathbf{y}) \in C_{0}^{\infty}(\Omega), \quad N \in \mathbb{N} .
$$

Положим также

$$
N(t)=\max \left\{N \in \mathbb{N}: \frac{N}{\lambda(N)}<t\right\} .
$$

При $t \in[0,1 / \lambda(1)]$ считаем, что $N(t) \equiv 0$. Очевидно, функция $N(t)$ является кусочно постоянной неубываюшей функцией при $t \geqslant 0$.

Справедливо следующее утверждение. 
ТЕОРема 1. Пусть выполнены условия (0.9)-(0.11). Тогда существует $\lambda$-последовательность и найдутся такие положительные постоянные $M, \kappa$, зависящие только от $k, q, \theta, \widehat{a}, \bar{a}, n$, что для решения $и(t, x, \mathbf{y})$ задачи $(0.1)-(0.3)$ при $t>2 / \lambda(2)$ справедливо неравенство

$$
\|u(t)\| \leqslant M \exp (-\kappa N(t))\|\varphi\| .
$$

Конечно, оценка (0.14) зависит от выбора $\lambda$-последовательности. Назовем $\bar{\lambda}$-последовательность оптимальной, если существует постоянная $C(k, q, \theta, \widehat{a}, \bar{a}, n)$ такая, что для любой другой $\lambda$-последовательности при всех достаточно больших $t$ вьполнено неравенство $N(t) \leqslant C \bar{N}(t)$. Здесь и далее элементы, относящиеся к оптимальной $\bar{\lambda}$-последовательности, будем помечать чертой сверху. В $\S 3$ мы покажем, что оптимальной является $\lambda$-последовательность с минимально возможными, без нарушения условия $(0.8)$, интервалами $\left(x_{j}, x_{j+1}\right)$ (следствие 2$)$.

В примерах будем требовать выполнение следуюшего условия. Пусть существует номер $s \in \overline{l, m}$ и постоянная $\widehat{b}>0$ такие, что для п.в. $x \in \mathbb{R}$ и любой функции $g(x, \mathbf{y}) \in C_{0}^{\infty}(\Omega)$ справедливо неравенство

$$
\int_{\mathbb{R}_{n}} B(g) d \mathbf{y} \geqslant \widehat{b} \int_{\mathbb{R}_{n}}\left\{\left(D_{y_{1}}^{s} g\right)^{2}+\left(D_{y_{1}}^{m} g\right)^{2}\right\} d \mathbf{y} .
$$

Отметим, что для коэффициентов $b_{\alpha \beta}(x)$, зависяших только от $x$, неравенство

$$
\sum_{i=l}^{m} \sum_{|\alpha|=|\beta|=i} b_{\alpha \beta}(x) \mathbf{z}^{\alpha} \mathbf{z}^{\beta} \geqslant \widehat{b}\left(z_{1}^{2 s}+z_{1}^{2 m}\right),
$$

справедливое при п.в. $x \in \mathbb{R}$ и любых $\mathbf{z} \in \mathbb{R}_{n}$, гарантирует выполнение (0.15). В случае коэффициентов, зависящих и от $\mathbf{y}$, для выполнения (0.15) достаточно справедливости неравенства

$$
\sum_{i=l}^{m} \sum_{|\alpha|=|\beta|=i} b_{\alpha \beta}(x, \mathbf{y}) z_{\alpha} z_{\beta} \geqslant \widehat{b}\left\{z_{s_{1}}^{2}+z_{m_{1}}^{2}\right\}
$$

для п.в. $(x, \mathbf{y}) \in \Omega$ и любого набора чисел $z_{\alpha}$; здесь $s_{1}=(s, 0, \ldots, 0), m_{1}=$ $(m, 0, \ldots, 0)$ - мультииндексы.

В качестве первого примера рассмотрим следуюшую область. Пусть $\left\{x_{j}\right\}_{j=0}^{\infty}$ - последовательность положительных чисел такая, что $x_{j+1}-x_{j} \geqslant 3, j=\overline{0, \infty}$. Пусть $\mathbb{R}_{2}^{+}=\left\{\left(x, y_{1}\right) \in \mathbb{R}_{2}: x>0\right\}$ - полуплоскость и

$$
\Omega=\mathbb{R}_{2}^{+} \backslash\left(\bigcup_{j=0}^{\infty} \bigcup_{i=-\infty}^{\infty} B\left(a ;\left(x_{j}+1, i\right)\right)\right), \quad a \in\left(0, \frac{1}{2}\right) .
$$

В силу неравенства $(0.15)$ и периодичности в расположении кругов при всех $j=$ $\overline{0, \infty}$ выполнено неравенство $\lambda\left(x_{j}, x_{j}+2\right) \geqslant \mathrm{const}>0$. Тогда из неравенства $(3.5)$ с $z=a=x_{j}, b=x_{j}+2, c=x_{j+1}, i=q$ следует, что $\left\{x_{j}\right\}_{j=0}^{\infty}$ является $\lambda$-последовательностью и справедлива оценка (0.14). 
Приведем другое следствие оценки (0.14).

Определим область типа слоя

$$
\Omega[f]=\left\{(x, \mathbf{y}) \in \mathbb{R}_{n+1}: x>d,\left|y_{1}\right|<f(x)\right\}
$$

с положительной непрерьвной функцией $f(x)(d \in(-\infty, 0])$. Будем предполагать, что функция $f$ удовлетворяет условию

$$
\int_{x_{0}}^{\infty} \frac{d x}{f^{[m / k, s / q]}(x)}=\infty
$$

Введем обозначение

$$
P^{s}(\rho, z)=\left\{\left(x, y_{1}\right) \in \mathbb{R}_{2}: z<x<z+\rho, 0<y_{1}<\rho^{[k / m, q / s]}\right\}
$$

для прямоугольника ширины $\rho$ с левой нижней вершиной в точке $z$ оси абсцисс. Символ $\Gamma_{a}^{b}(f)$ будет обозначать криволинейную трапецию

$$
\Gamma_{a}^{b}(f)=\left\{\left(x, y_{1}\right) \in \mathbb{R}_{2}: a<x<b, 0<y_{1}<f(x)\right\},
$$

причем параметр $b=\infty$ может быть опушен. Пусть $\rho_{*}(r)$ - ширина наибольшего прямоугольника $P^{s}\left(\rho_{*}, z_{*}\right)$, содержашегося в $\Gamma_{x_{0}}^{r}(f)$.

Чтобы выразить оценку (0.14) для областей вида $(0.16)$ в терминах функции $f(x)$, определим понятие П-последовательности. Неограниченную возрастающую последовательность положительных чисел $\left\{x_{j}\right\}_{j=0}^{\infty}$ назовем $\Pi^{s}$-последовательностью, если справедливы равенства

$$
\min \left\{f^{[m, s]}(x): x \in\left[x_{j}, x_{j+1}\right]\right\}=\left(x_{j+1}-x_{j}\right)^{[k, q]}, \quad j=\overline{0, \infty} .
$$

Отметим, что $\Pi^{s}$-последовательность можно построить всегда, начиная с произвольной точки $x_{0}>0$. Действительно, выберем $\rho$ максимально возможным такое, что прямоугольник $P^{s}\left(\rho, x_{0}\right) \subset \Gamma_{x_{0}}(f)$. Положим $x_{1}=x_{0}+\rho$, тогда, очевидно, $\min _{\left[x_{0}, x_{1}\right]} f^{[m, s]}(x)=\rho^{[k, q]}=\left(x_{1}-x_{0}\right)^{[k, q]}$. Таким же образом можно построить и остальные элементы $\Pi^{s}$-последовательности. В утверждении 5 доказывается, что если выполнено условие (0.15), то П-последовательность является $\lambda$-последовательностью.

Определим функцию $r(t), t \geqslant 0$, равенством

$$
\int_{x_{0}}^{r} \frac{d x}{f^{[m / k, s / q]}(x)}=\frac{t}{\rho_{*}^{2[k, q]}(r)} .
$$

Ввиду возрастания функции

$$
\rho_{*}^{2[k, q]}(r) \int_{x_{0}}^{r} \frac{d x}{f^{[m / k, s / q]}(x)}, \quad r \geqslant x_{0},
$$

равенство (0.19) однозначно определяет монотонно возрастаюшую функцию $r(t)$, $t \geqslant 0$. 
Для областей $\Omega[f]$ типа слоя (0.16) в $§ 4$ получено следствие оценки $(0.14)$ (см. утверждение 6):

$$
\|u(t)\|_{\Omega[f]} \leqslant M_{1} \exp \left(-\kappa_{1} \int_{x_{0}}^{r(t)} \frac{d x}{f^{[m / k, s / q]}(x)}\right)\|\varphi\|_{\Omega[f]}, \quad t \geqslant T_{1} .
$$

Если существует постоянная $c \geqslant 1$ такая, что

$$
\max \left\{f(z): z \in\left[x-f^{[m / k, l / q]}(x), x+f^{[m / k, l / q]}(x)\right]\right\} \leqslant c f(x), \quad x \geqslant x_{0},
$$

то (0.20) при $s=l$ устанавливает такой же порядок убывания решения, что и оценка (0.14) с оптимальной $\bar{\lambda}$-последовательностью (утверждение 7$)$. В частности, для функции $f(r)=r^{a}, a<q / s, r>0$, оценка (0.20) приобретает вид

$$
\|u(t)\|_{\Omega[f]} \leqslant M_{1} \exp \left(-\kappa_{1} t^{b}\right)\|\varphi\|_{\Omega[f]}, \quad b=\frac{q-s a}{q-s a+2 s a q} .
$$

В случае параболического уравнения второго порядка для одного класса областей в $\S 5$ получена оценка снизу неотрицательного решения задачи, подтверж даюшая точность оценки $(0.14)$.

Определим область в $\mathbb{R}_{n+1}$, которую будем назьвать модельной трубкой. Пусть ломаная $\Gamma$, соединяюшая точки $(a / 2, \mathbf{0})$ и $(1-a / 2, \mathbf{0}), a \in(0,1 / 2)$, такова, что ее $\varepsilon$-окрестность $U(\Gamma)$ лежит в слое $\Pi_{0}^{1}=\left\{(x, \mathbf{y}) \in \mathbb{R}_{n+1}: 0<x<1\right\}$. Модельной трубкой $M$ назовем односвязную область вида

$$
M=\Pi_{0}^{1} \cap(U(\Gamma) \cup B(a ;(0, \mathbf{0})) \cup B(a ;(1, \mathbf{0}))) .
$$

ТеОрема 2. Пусть $k=q=m=l=1$ и уравнение (0.1) является равномерно параболическим уравнением второго порядка. Пусть область $\Omega$ и $\lambda$-последовательность $\left\{x_{j}\right\}_{j=0}^{\infty}$ таковы, что при всех $j=\overline{0, \infty}$ выполнены включения

$$
\left(x_{j}, \mathbf{0}\right)+\left(x_{j+1}-x_{j}\right) \bar{M} \subset \Omega,
$$

где левую часть следует понимать как гомотетию и параллельный перенос, примененные $\kappa$ замыканию $\bar{M}$. Пусть справедливы неравенства

$$
\frac{1}{b} \leqslant \frac{x_{j+2}-x_{j+1}}{x_{j+1}-x_{j}} \leqslant b, \quad j=\overline{0, \infty}, \quad b \geqslant 1 .
$$

Тогда при выполнении условия (0.11) справедлива оченка (0.14) для неотрицательного решения задачи (0.1)-(0.3) с неотрицательной финитной начальной функцией $\varphi(x, \mathbf{y}) \not \equiv 0$, и она является точной. А именно существуют положительнье постоянные $\kappa_{0}, M_{0}$, зависящие только от $k, q, \theta, \widehat{a}, \bar{a}, n, \widehat{b}$, $R_{0}, \Omega, \varphi$, такие, что для решения и $(t, x, \mathbf{y})$ при

$$
t>\max \left\{\frac{a^{2}\left(x_{1}-R_{0}\right)^{2}}{64}, \frac{2}{\lambda(2)}\right\}
$$


справедливы неравенства

$$
M_{0} \exp \left(-\kappa_{0} N(t)\right) \leqslant\|u(t)\| \leqslant M \exp (-\kappa N(t))\|\varphi\| .
$$

Из этой теоремы следует, что оценка $(0.20)$ с функцией $f$, удовлетворяющей условию (0.21), является точной при $k=q=m=l=1$ (см. $\S 5)$.

Приведем еще один класс примеров, для которых оценка (0.14) решения параболического уравнения второго порядка точна. Пусть $Q \subset \bar{\Pi}_{0}^{1}$ - такое замкнутое множество, что $\Pi_{0}^{1} \backslash Q$ содержит модельную трубку $M$ и

$$
\lambda=\inf \left\{\int_{\Pi_{0}^{1} \backslash Q} F(g) d x d \mathbf{y}: g \in C_{0}^{\infty}\left(\mathbb{R}_{n+1}\right),\left.g\right|_{Q}=0, \int_{\Pi_{0}^{1} \backslash Q} g^{2} d x d \mathbf{y}=1\right\}>0 .
$$

Пусть $\left\{x_{j}\right\}_{j=0}^{\infty}-$ некоторая последовательность положительных чисел, удовлетворяющая условию (0.24). Пусть область $\Omega$, удовлетворяющая условию $(0.11)$, такова, что при $j=\overline{0, \infty}$ выполнены равенства

$$
\Omega_{x_{j}}^{x_{j+1}}=\left(x_{j}, \mathbf{0}\right)+\left(x_{j+1}-x_{j}\right)\left(\Pi_{0}^{1} \backslash Q\right)
$$

и включения (0.23). Тогда при $k=q=m=l=1\left\{x_{j}\right\}_{j=0}^{\infty}$ является $\lambda$-последовательностью, справедлива оценка (0.14) и она точна.

\section{§1. Вспомогательные утверждения}

Гильбертово пространство $\stackrel{\circ}{W} \underset{F}{ }(\Omega)$ определим как пополнение пространства $C_{0}^{\infty}(\Omega)$ по норме

$$
\|g\|_{W_{F}^{\circ}(\Omega)}^{2}=\|g\|^{2}+\|F(g)\|_{L_{1}(\Omega)} .
$$

Пространства $\stackrel{\circ}{W} \underset{F}{0}\left(D^{T}\right), \stackrel{\circ}{W} \underset{F}{1}\left(D^{T}\right)$ определим как пополнения $C_{0}^{\infty}\left(D_{-1}^{T+1}\right)$ по нормам соответственно

$$
\begin{aligned}
\|u\|_{W_{F}^{0}\left(D^{T}\right)}^{2} & =\int_{0}^{T}\left(\|u(t)\|^{2}+\|F(u)(t)\|_{L_{1}(\Omega)}\right) d t, \\
\|u\|_{W_{F}^{1}\left(D^{T}\right)}^{2} & =\int_{0}^{T}\left(\|u(t)\|^{2}+\left\|u_{t}(t)\right\|^{2}+\|F(u)(t)\|_{L_{1}(\Omega)}\right) d t .
\end{aligned}
$$

Обобщенным решением задачи (0.1)-(0.3) в $D^{T}$ назовем функцию $u(t, x, \mathbf{y}) \in$ $\stackrel{\circ}{W}_{F}^{0}\left(D^{T}\right)$, удовлетворяющую интегральному тождеству

$$
\begin{aligned}
\int_{D^{T}} & {\left[-u v_{t}+\sum_{i=q}^{k} a_{i}(x, \mathbf{y}) D_{x}^{i} u D_{x}^{i} v+\sum_{i=l}^{m} \sum_{|\alpha|=|\beta|=i} b_{\alpha \beta}(x, \mathbf{y}) D_{\mathbf{y}}^{\alpha} u D_{\mathbf{y}}^{\beta} v\right] d x d \mathbf{y} d t } \\
= & \int_{\Omega} \varphi(x, \mathbf{y}) v(0, x, \mathbf{y}) d x d \mathbf{y}
\end{aligned}
$$

для любой функции $v(t, x, \mathbf{y}) \in \stackrel{\circ}{W_{F}^{1}}{ }_{F}\left(D^{T}\right), v(T, x, \mathbf{y})=0$. Функцию $u(t, x, \mathbf{y})$ будем называть решением задачи (0.1)-(0.3) в $D$, если при всех $T>0$ она является решением этой задачи в $D^{T}$. 
Обобщенное решение задачи (0.1)-(0.3) существует и единственно. Существование доказывается методом галёркинских приближений аналогично доказательству для уравнения второго порядка [13; гл. III, § 4, теорема 4.1]. Единственность вытекает из следующего утверждения.

УТВЕРЖДЕНИЕ 1. Обобщенное решение $u(t, x, \mathbf{y})$ задачи $(0.1)-(0.3)$ принадлежит классу $C\left([0, \infty) \rightarrow L_{2}(\Omega)\right)$ и при всех $t \geqslant 0$ удовлетворяет равенству

$$
\|u(t)\|^{2}+2 \int_{0}^{t}\|F(u)(\tau)\|_{L_{1}(\Omega)} d \tau=\|\varphi\|^{2} .
$$

Доказательство этого утверждения проводится так же, как для линейного параболического уравнения второго порядка [13; гл. III, $\S 2$, теорема 2.1].

Лемма 1. Для любой функиии $g(x) \in C^{\infty}[a, b]$ и любого $\varepsilon \leqslant(b-a)^{2}$ справедливо неравенство

$$
\int_{a}^{b} g_{p}^{2} d x \leqslant \varepsilon^{-p} \int_{a}^{b}\left[\varepsilon^{i} g_{i}^{2}+G_{i} g^{2}\right] d x, \quad p=\overline{0, i-1}
$$

әде $g_{p}(x)=D_{x}^{p} g(x), G_{i} \geqslant 1$ - постоянная, зависящая только от $i$. Здесь $i$ произвольное натуральное число.

ДоКАЗАТЕЛЬСТво достаточно провести для отрезка $[0,1]$. Общий случай сводится к этому заменой $x=a+(b-a) y$.

Для функции $g(x) \in C_{0}^{\infty}(0,1)$ справедливы разложение в ряд Фурье

$$
g(x)=\sum_{k=1}^{\infty} a_{k} \sin k \pi x
$$

и равенства Парсеваля

$$
\left\|g_{p}\right\|^{2} \equiv \int_{0}^{1} g_{p}^{2} d x=C \pi^{2 p} \sum_{k=1}^{\infty} k^{2 p} a_{k}^{2}, \quad p=\overline{0, i}
$$

Применив неравенство Юнга для $p=\overline{1, i-1}$

$$
\pi^{2 p} k^{2 p} \leqslant \frac{p}{i} \varepsilon_{1}^{i-p} k^{2 i} \pi^{2 i}+\frac{i-p}{i} \varepsilon_{1}^{-p}
$$

получим

$$
\left\|g_{p}\right\|^{2} \leqslant \varepsilon_{1}^{-p}\left(\varepsilon_{1}^{i}\left\|g_{i}\right\|^{2}+\|g\|^{2}\right) \quad \forall \varepsilon_{1}>0
$$

Для $p=0$ неравенство очевидно, поэтому (1.4) справедливо для $p=\overline{0, i-1}$.

Проведем теперь индукцией по $i$ доказательство неравенств (1.3) для произвольной функции $g \in C^{\infty}[0,1]$. При $i=1, p=0$ неравенство (1.3) очевидно. Пусть неравенства (1.3) справедливы до порядка $i$. Докажем в этом предположении, что

$$
\left\|g_{i}\right\|^{2} \leqslant \varepsilon^{-i}\left(\varepsilon^{i+1}\left\|g_{i+1}\right\|^{2}+G_{i+1}\|g\|^{2}\right), \quad \varepsilon \leqslant 1 .
$$


Возьмем продолжение $\widetilde{g} \in C^{\infty}[-1,2]$ такое, что $\left.\widetilde{g}\right|_{[0,1]}=g$ и

$$
\int_{-1}^{2} \widetilde{g}_{p}^{2} d x \leqslant C(i) \int_{0}^{1} g_{p}^{2} d x, \quad p=\overline{0, i+1}
$$

где постоянная $C(i)$ зависит только от $i$.

Пусть $\eta(x) \in C_{0}^{\infty}(-1,2)$ - срезаюшая функция, не превосходящая единицы, равная единице на $[0,1]$. Тогда $v=\widetilde{g} \eta \in C_{0}^{\infty}(-1,2)$ и из (1.4) следует, что

$$
\int_{-1}^{2} v_{i}^{2} d x \leqslant \varepsilon_{2} \int_{-1}^{2} v_{i+1}^{2} d x+\varepsilon_{2}^{-i} \int_{-1}^{2} v^{2} d x, \quad \varepsilon_{2}>0
$$

Поскольку ввиду (1.5)

$$
\int_{-1}^{2} v_{i+1}^{2} d x \leqslant C_{i} \sum_{p=0}^{i+1} \int_{-1}^{2} \tilde{g}_{p}^{2} d x \leqslant C_{i} C(i) \sum_{p=0}^{i+1} \int_{0}^{1} g_{p}^{2} d x
$$

то из (1.6) следует неравенство

$$
\left\|g_{i}\right\|^{2} \leqslant \varepsilon_{2} C(i) C_{i} \sum_{p=0}^{i+1}\left\|g_{p}\right\|^{2}+\varepsilon_{2}^{-i} C(i)\|g\|^{2}, \quad \varepsilon_{2}>0
$$

Сложив при $\varepsilon=1$ неравенства индуктивного предположения (1.3), устанавливаем, что

$$
\sum_{p=1}^{i-1}\left\|g_{p}\right\|^{2} \leqslant(i-1)\left\|g_{i}\right\|^{2}+(i-1) G_{i}\|g\|^{2}
$$

поэтому

$$
\left\|g_{i}\right\|^{2} \leqslant \varepsilon_{2} C(i) C_{i}\left(\left\|g_{i+1}\right\|^{2}+i\left\|g_{i}\right\|^{2}+i G_{i}\|g\|^{2}\right)+\varepsilon_{2}^{-i} C(i)\|g\|^{2}, \quad \varepsilon_{2}>0
$$

Выбирая $\varepsilon_{2}$ так, чтобы $\varepsilon_{2} C(i) C_{i} i \leqslant \delta<1$, замечаем, что

$$
\left\|g_{i}\right\|^{2} \leqslant \frac{\varepsilon_{2} C(i) C_{i}}{1-\delta}\left\|g_{i+1}\right\|^{2}+\frac{\varepsilon_{2} C(i) i C_{i} G_{i}+\varepsilon_{2}^{-i} C(i)}{1-\delta}\|g\|^{2} .
$$

Полагая $\varepsilon=\varepsilon_{2} C(i) C_{i} /(1-\delta)$, находим, что $\varepsilon \in(0, \delta /(i(1-\delta))] \equiv(0,1]$ при подходящем выборе $\delta$. Отсюда

$$
\left\|g_{i}\right\|^{2} \leqslant \varepsilon\left\|g_{i+1}\right\|^{2}+G_{i+1} \varepsilon^{-i}\|g\|^{2} .
$$

Производные меньшего порядка оцениваются при помощи (1.3). Лемма доказана. 


\section{§2. Оценка решения при больших $x$}

Доказательство теоремы 1 опирается на оценку решения при больших $x$, устанавливаемую в нижеследуюшей теореме 3 .

Ниже через $\Xi=\{k, q, \theta, \widehat{a}, \bar{a}, n\}$ будем обозначать набор постоянных. Зависимость других постоянных от этого набора будем указывать в круглых скобках.

ТЕОРема 3. Пусть выполнены условия (0.9)-(0.11). Тогда существует $\lambda$-последовательность и найдутся положительные постоянные $\kappa(\Xi), \widetilde{M}(\Xi)$ такие, что решение $и(t, x, \mathbf{y})$ задачи $(0.1)-(0.3)$ при всех $t \geqslant 0$ и $N \geqslant 2$ удовлетворяет оценке

$$
\|u(t)\|_{\Omega_{x_{N}}}^{2} \leqslant \widetilde{M} \exp (-2 \kappa N)\|\varphi\|^{2} .
$$

ДоказАТЕЛЬСтво. Построим срезаюшую функцию $\xi(x), x \in \mathbb{R}$. Пусть $h(t) \in$ $C^{\infty}[0,1]$ - неубываюшая функция, равная 0 и 1 в окрестности нуля и единицы соответственно. Пусть

$$
a_{i}=3^{i} \max _{t \in[0,1]}\left|D^{i} h(t)\right|, \quad i=\overline{0, \infty}
$$

Из теоремы Лагранжа следует, что $a_{i+1} \geqslant a_{i}$.

Определим дифференциальные полиномы $P_{p}$ от гладкой функции $\alpha(x)$ условиями $P_{0}=1, P_{p}=(D+2 \alpha) P_{p-1}$. Тогда

$$
\begin{gathered}
P_{p}=(D+2 \alpha)^{p-1} 2 \alpha=\sum_{s=1}^{p} A_{s p}^{p_{1} p_{2} \ldots p_{s}} \prod_{\substack{p_{1}+2 p_{2}+\cdots+s p_{s}=p \\
p=\overline{1, \infty}}} \alpha^{p_{1}(D \alpha)^{p_{2}}\left(D^{s-1} \alpha\right)^{p_{s}}} \\
\end{gathered}
$$

где $A_{s p}^{p_{1} p_{2} \ldots p_{s}}$ - целые неотрицательные числа.

Положим

$$
b_{p}=\sum_{s=1}^{p} A_{s p}^{p_{1} p_{2} \ldots p_{s}} \prod_{p_{1}+2 p_{2}+\cdots+s p_{s}=p} a_{0}^{p_{1}} a_{1}^{p_{2}} \cdots a_{s-1}^{p_{s}}
$$

очевидно, $b_{p+1} \geqslant b_{p}$.

Выберем число $e_{*}>1$ так, чтобы вьполнялось неравенство

$$
\exp \left(\frac{2}{e_{*}}\right)\left(\frac{\widetilde{b}}{e_{*}}\right)^{2} k\left\{1+(k-q+1) \widehat{a} \max _{i=\overline{q, k}} G_{i} \theta\right\} \leqslant 1,
$$

где $\widetilde{b}=\frac{\bar{a}}{\widehat{a}} 2^{k} b_{k}, G_{i}-$ константы из неравенства (1.3).

Зафиксируем натуральное число $N \geqslant 2$. Рассмотрим кусочно постоянную функцию $\beta(x)$ на промежутке $\left[x_{0}, x_{N+1}\right)$ такую, что $\beta(x)=\delta_{j}, x \in\left[x_{j}, x_{j+1}\right)$, $j=\overline{0, N}$, причем $\delta_{0}=\delta_{N}=0$ и $\delta_{j}^{-1}=e_{*} \Delta_{j}, j=\overline{1, N-1}$, где $\Delta_{j}=\left(x_{j+1}-x_{j}\right)$. 
Построим функцию $\alpha(x) \leqslant \beta(x)$, сглаживая функцию $\beta(x)$ по следующему правилу. Если $\delta_{j-1}<\delta_{j}$, то на отрезке $\left[x_{j}, x_{j}+\Delta_{j} / 3\right]$ функция $\alpha(x)$ определяется так:

$$
\alpha(x)=\delta_{j-1}+\left(\delta_{j}-\delta_{j-1}\right) h\left(\frac{3\left(x-x_{j}\right)}{\Delta_{j}}\right) .
$$

Если же $\delta_{j}>\delta_{j+1}$, то на отрезке $\left[x_{j+1}-\Delta_{j} / 3, x_{j+1}\right]$ полагаем

$$
\alpha(x)=\delta_{j}-\left(\delta_{j}-\delta_{j+1}\right) h\left(1+\frac{3\left(x-x_{j+1}\right)}{\Delta_{j}}\right) .
$$

В оставшихся точках полагаем $\alpha(x)=\beta(x)$.

Очевидно, что во всех случаях производные функции $\alpha(x)$ подчиняются оценкам

$$
\left|D^{i} \alpha(x)\right| \leqslant\left(\frac{3}{\Delta_{j}}\right)^{i} \delta_{j} \max _{t \in[0,1]}\left|D^{i} h(t)\right| \leqslant \frac{a_{i} \delta_{j}}{\Delta_{j}^{i}}=\frac{a_{i}}{e_{*} \Delta_{j}^{i+1}}
$$

при всех $x \in\left[x_{j}, x_{j+1}\right], j=\overline{1, N-1}, i \in \mathbb{N}$. Следовательно, полиномы $P_{p}$ удовлетворяют неравенствам

$$
\begin{aligned}
& \left|P_{p}\right| \leqslant \sum_{s=1}^{p} A_{s p}^{p_{1} p_{2} \ldots p_{s}} \prod_{p_{1}+2 p_{2}+\cdots+s p_{s}=p}\left(\frac{a_{0}}{e_{*} \Delta_{j}}\right)^{p_{1}} \cdots\left(\frac{a_{s-1}}{e_{*} \Delta_{j}^{s}}\right)^{p_{s}} \leqslant \frac{b_{p}}{e_{*} \Delta_{j}^{p}}, \\
& x \in\left[x_{j}, x_{j+1}\right], \quad j=\overline{1, N-1}, \quad p \in \mathbb{N} \text {. }
\end{aligned}
$$

Установим двусторонние оценки интеграла $\int_{x_{j}}^{x_{j+1}} \alpha(t) d t, j=\overline{1, N-1}$ :

$$
\frac{1}{3 e_{*}}=\frac{\delta_{j} \Delta_{j}}{3}=\int_{x_{j}+\Delta_{j} / 3}^{x_{j}+2 \Delta_{j} / 3} \delta_{j} d t \leqslant \int_{x_{j}}^{x_{j+1}} \alpha(t) d t \leqslant \int_{x_{j}}^{x_{j+1}} \delta_{j} d t=\delta_{j} \Delta_{j}=\frac{1}{e_{*}} .
$$

Определим неубываюшую гладкую функцию $\xi(x)$ на $\mathbb{R}$ условиями

$$
\xi(x)= \begin{cases}0, & x \leqslant x_{0} \\ \xi\left(x_{1}\right) h^{1 / 2}\left(\frac{3\left(x-x_{0}\right)}{\Delta_{0}}\right), & x_{0} \leqslant x \leqslant x_{0}+\frac{\Delta_{0}}{3} \\ \exp \left(-\int_{x}^{x_{N}} \alpha(t) d t\right), & x_{0}+\frac{\Delta_{0}}{3} \leqslant x \leqslant x_{N} \\ 1, & x \geqslant x_{N} .\end{cases}
$$

На промежутке $\left[x_{1}, x_{N}\right]$ справедливо уравнение $\xi^{\prime}=\alpha \xi$ и ввиду (2.7) справедливы неравенства

$$
\exp \left(\frac{1}{3 e_{*}}\right) \leqslant \frac{\xi\left(x_{j+1}\right)}{\xi\left(x_{j}\right)} \leqslant \exp \left(\frac{1}{e_{*}}\right), \quad j=\overline{1, N-1}
$$


Перемножая эти неравенства, находим, что $\exp \left(\frac{N-1}{3 e_{*}}\right) \leqslant \frac{\xi\left(x_{N}\right)}{\xi\left(x_{1}\right)}$ и

$$
\xi\left(x_{1}\right) \leqslant \exp \left(-\frac{N-1}{3 e_{*}}\right)
$$

Нетрудно доказать, что на промежутке $\left[x_{1}, x_{N}\right]$ справедливо соотношение

$$
D^{p} \xi^{2}=\xi^{2} P_{p}, \quad p \in \mathbb{N} .
$$

Из определения функций $\alpha(x), \xi(x)$ следует, что

$$
\xi(x)=\xi\left(x_{1}\right), \quad x \in\left[x_{0}+\frac{\Delta_{0}}{3}, x_{1}\right] .
$$

Оценим производные $D^{p} \xi^{2}$ на отрезке $\left[x_{0}, x_{1}\right]$ :

$$
\left|D^{p} \xi^{2}\right| \leqslant \xi^{2}\left(x_{1}\right)\left|D^{p} h\left(\frac{3\left(x-x_{0}\right)}{\Delta_{0}}\right)\right| \leqslant \frac{a_{p}}{\Delta_{0}^{p}} \xi^{2}\left(x_{1}\right) .
$$

Через $f_{h}(t)$ обозначим осреднение Стеклова $f_{h}(t)=\frac{1}{h} \int_{t}^{t+h} f(\tau) d \tau$. В тождестве (1.1) возьмем функцию $v=\omega_{-h}, 0<h \leqslant \delta, \omega \in C_{0}^{\infty}\left(D^{T-\delta}\right)$. После простых преобразований придем к тождеству

$$
\begin{aligned}
& \int_{D^{T-\delta}}\left\{\left(u_{h}\right)_{t} \omega+\sum_{i=q}^{k} a_{i}(x, \mathbf{y}) D_{x}^{i} u_{h} D_{x}^{i} \omega\right. \\
& \left.+\sum_{i=l}^{m} \sum_{|\alpha|=|\beta|=i} b_{\alpha \beta}(x, \mathbf{y}) D_{\mathbf{y}}^{\alpha} u_{h} D_{\mathbf{y}}^{\beta} \omega\right\} d x d \mathbf{y} d t=0
\end{aligned}
$$

которое справедливо также для $\omega \in \stackrel{\circ}{W_{F}^{0}}\left(D^{T-\delta}\right)$. Далее, ввиду произвольности $T>0$ можем считать, что (2.12) справедливо в $D^{t}, t>0$, при $\omega \in \stackrel{\circ}{W_{F}^{0}}\left(D^{t}\right)$.

Положим в $(2.12) \omega=u_{h} \xi^{2}(x) \in \stackrel{\circ}{W_{F}^{0}}\left(D^{t}\right)$ :

$$
\begin{aligned}
& \int_{\Omega}\left(u_{h} \xi\right)^{2}(t) d x d \mathbf{y}+2 \int_{D^{t}}\left\{\sum_{i=q}^{k} a_{i}(x, \mathbf{y}) D_{x}^{i} u_{h} D_{x}^{i}\left(u_{h} \xi^{2}\right)+\xi^{2} B\left(u_{h}\right)\right\} d x d \mathbf{y} d \tau \\
& =\int_{\Omega}\left(u_{h} \xi\right)^{2}(0) d x d \mathbf{y} .
\end{aligned}
$$

После предельного перехода при $h \rightarrow 0$ получаем (с учетом того, что $\xi \varphi \equiv 0)$

$$
\begin{aligned}
& \int_{\Omega}(u \xi)^{2}(t) d x d \mathbf{y}+2 \int_{D^{t}} F(u) \xi^{2} d x d \mathbf{y} d \tau \\
& \quad=-2 \sum_{i=q}^{k} \int_{D^{t}} a_{i}(x, \mathbf{y}) D_{x}^{i} u\left(\sum_{p=1}^{i} C_{i}^{p} D^{p}\left(\xi^{2}\right) D_{x}^{i-p} u\right) d x d \mathbf{y} d \tau .
\end{aligned}
$$


Оценим правую часть с учетом (2.10) и того, что $D_{x}\left(\xi^{2}\right) \equiv 0$ вне промежутка $\left[x_{0}, x_{N}\right]$. На промежутке $\left[x_{j}, x_{j+1}\right], j=\overline{1, N-1}$, рассмотрим интеграл

$$
I_{j} \equiv 2 \bar{a} \sum_{i=q}^{k} \int_{0}^{t} \int_{\Omega_{x_{j}}^{x_{j}+1}} \xi^{2}\left|D_{x}^{i} u\right| \sum_{p=1}^{i} C_{i}^{p}\left|P_{p}\right|\left|D_{x}^{i-p} u\right| d x d \mathbf{y} d \tau
$$

Согласно (2.6) имеем

$$
\begin{aligned}
I_{j} \leqslant & 2 \bar{a} \sum_{i=q}^{k} \int_{0}^{t} \int_{\Omega_{x_{j}}^{x_{j+1}}} \xi^{2}\left|D_{x}^{i} u\right| \sum_{p=1}^{i} \frac{C_{i}^{p} b_{p}}{e_{*} \Delta_{j}^{p}}\left|D_{x}^{i-p} u\right| d x d \mathbf{y} d \tau \\
\leqslant & \widehat{a} \sum_{i=q}^{k} \int_{0}^{t} \int_{\Omega_{x_{j}}^{x_{j+1}}} \xi^{2}\left(D_{x}^{i} u\right)^{2} d x d \mathbf{y} d \tau \\
& +\frac{\bar{a}^{2}}{\widehat{a}} \sum_{i=q}^{k} \int_{0}^{t} \int_{\Omega_{x_{j}}^{x_{j+1}}} \xi^{2}\left(\sum_{p=1}^{i} \frac{C_{i}^{p} b_{p}}{e_{*} \Delta_{j}^{p}}\left|D_{x}^{i-p} u\right|\right)^{2} d x d \mathbf{y} d \tau \\
\equiv & \widehat{I}_{j}+\bar{I}_{j} .
\end{aligned}
$$

Применив ко второму слагаемому неравенство Коши-Буняковского, пользуясь леммой 1 с $\varepsilon=\Delta_{j}^{2}$, находим, что

$$
\begin{aligned}
\bar{I}_{j} & \leqslant \frac{\bar{a}^{2}}{\widehat{a}} \xi^{2}\left(x_{j+1}\right)\left(\frac{2^{k} b_{k}}{e_{*}}\right)^{2} \sum_{i=q}^{k} \int_{0}^{t} \int_{\Omega_{x_{j}}} \sum_{p=1}^{i} \frac{\left|D_{x}^{i-p} u\right|^{2}}{\Delta_{j}^{2 p}} d x d \mathbf{y} d \tau \\
& \leqslant\left(\frac{\widetilde{b}}{e_{*}}\right)^{2} \widehat{a} \xi^{2}\left(x_{j+1}\right) \sum_{i=q}^{k} \int_{0}^{t} \int_{\Omega_{x_{j}} x_{j+1}} i\left[\left(D_{x}^{i} u\right)^{2}+G_{i} \frac{u^{2}}{\Delta_{j}^{2 i}}\right] d x d \mathbf{y} d \tau .
\end{aligned}
$$

Далее, применив соотношение (2.8) и определение $\lambda$-последовательности $(0.8),(0.7)$, установим

$$
\begin{aligned}
\bar{I}_{j} \leqslant & \left(\frac{\widetilde{b}}{e_{*}}\right)^{2} \widehat{a} \exp \left(\frac{2}{e_{*}}\right) \xi^{2}\left(x_{j}\right) \sum_{i=q}^{k} \int_{0}^{t} \int_{\Omega_{x_{j}} x_{j+1}} i\left[\left(D_{x}^{i} u\right)^{2}\right. \\
& \left.+G_{i} \lambda^{-1}\left(x_{j}, x_{j+1}\right) \frac{F(u)}{\Delta_{j}^{2[k, q]}}\right] d x d \mathbf{y} d \tau \\
\leqslant & \left(\frac{\widetilde{b}}{e_{*}}\right)^{2} \exp \left(\frac{2}{e_{*}}\right) k \int_{0}^{t} \int_{\Omega_{x_{j}}^{x_{j+1}}}\left[A(u)+(k-q+1) \widehat{a} \max _{i=\overline{q, k}} G_{i} \theta F(u)\right] \xi^{2} d x d \mathbf{y} d \tau .
\end{aligned}
$$

Отсюда ввиду неравенств $(0.5),(2.4)$ получим

$$
I_{j} \leqslant 2 \int_{0}^{t} \int_{\Omega_{x_{j}}^{x_{j+1}}} F(u) \xi^{2} d x d \mathbf{y} d \tau, \quad j=\overline{1, N-1} .
$$


Теперь из (2.13) следует, что

$$
\begin{aligned}
& \int_{\Omega_{x_{N}}} u^{2}(t, x, \mathbf{y}) d x d \mathbf{y} \leqslant I_{0} \\
& \quad \equiv 2 \bar{a} \sum_{i=q}^{k} \int_{0}^{t} \int_{\Omega_{x_{0}}^{x_{1}}}\left|D_{x}^{i} u\right| \sum_{p=1}^{i} C_{i}^{p}\left|D^{p}\left(\xi^{2}\right)\right|\left|D_{x}^{i-p} u\right| d x d \mathbf{y} d \tau .
\end{aligned}
$$

Далее, согласно (2.11) имеем

$$
I_{0} \leqslant 2 \bar{a} \xi^{2}\left(x_{1}\right) \sum_{i=q}^{k} \int_{0}^{t} \int_{\Omega_{x_{0}}^{x_{1}}}\left|D_{x}^{i} u\right| \sum_{p=1}^{i} \frac{C_{i}^{p} a_{p}}{\Delta_{0}^{p}}\left|D_{x}^{i-p} u\right| d x d \mathbf{y} d \tau .
$$

Интеграл $I_{0}$ оценивается аналогично $I_{j}$ :

$$
I_{0} \leqslant \bar{a} \xi^{2}\left(x_{1}\right) \sum_{i=q}^{k} \int_{0}^{t} \int_{\Omega_{x_{0}}^{x_{1}}}\left[\left(1+\widetilde{a}^{2} i\right)\left|D_{x}^{i} u\right|^{2}+\frac{i G_{i} \widetilde{a}^{2}}{\Delta_{0}^{2 i}} u^{2}\right] d x d \mathbf{y} d \tau,
$$

где $\widetilde{a}=a_{k} 2^{k}$. Таким образом, ввиду $(0.7),(0.8),(0.5),(1.2)$ получим

где

$$
\begin{aligned}
I_{0} \leqslant & \bar{a} \xi^{2}\left(x_{1}\right) \int_{0}^{t} \int_{\Omega_{x_{0}}^{x_{1}}}\left[\frac{\left(1+\widetilde{a}^{2} k\right)}{\widehat{a}} A(u)\right. \\
& \left.+(k-q+1) \max _{i=\overline{q, k}} G_{i} \frac{k \widetilde{a}^{2} \lambda^{-1}\left(x_{0}, x_{1}\right)}{\Delta_{0}^{2[k, q]}} F(u)\right] d x d \mathbf{y} d \tau \\
\leqslant & 2 C \xi^{2}\left(x_{1}\right) \int_{0}^{t} \int_{\Omega_{x_{0}}^{x_{1}}} F(u) d x d \mathbf{y} d \tau \leqslant C \xi^{2}\left(x_{1}\right)\|\varphi\|^{2}
\end{aligned}
$$

$$
C=\frac{\bar{a}}{2 \widehat{a}}\left(1+\widetilde{a}^{2} k+(k-q+1) k \max _{i=\overline{q, k}} G_{i} \widetilde{a}^{2} \widehat{a} \theta\right) .
$$

Теперь оценка (2.1) следует из (2.14) и (2.9). Тем самым, установлено неравенство $(2.1)$ с $\kappa=1 /\left(3 e_{*}\right)<1 / 3$.

ДОКАЗАТЕЛЬСТВо ТЕОРЕМЫ 1 . ВЫберем Произвольное натуральное число $N \geqslant 2$. Из теоремы 3 имеем

$$
\int_{\Omega_{x_{N}}} u^{2}(t, x, \mathbf{y}) d x d \mathbf{y} \leqslant \widetilde{M} \exp (-2 \kappa N)\|\varphi\|^{2} \equiv \varepsilon(N)
$$

Следовательно, ввиду (0.12)

$$
\begin{aligned}
\int_{\Omega} u^{2}(t, x, \mathbf{y}) d x d \mathbf{y} & \leqslant \varepsilon(N)+\int_{\Omega^{x_{N}}} u^{2}(t, x, \mathbf{y}) d x d \mathbf{y} \\
& \leqslant \varepsilon(N)+\lambda^{-1}(N) \int_{\Omega^{x_{N}}} F(u) d x d \mathbf{y} .
\end{aligned}
$$

Теперь из (1.2) следует, что

$$
\|u(t)\|^{2} \leqslant \varepsilon(N)-\frac{\lambda^{-1}(N)}{2} \frac{d}{d t}\|u(t)\|^{2} .
$$

Решая это дифференциальное неравенство, будем иметь

$$
\|u(t)\|^{2} \leqslant \widetilde{M} \exp (-2 \kappa N)\|\varphi\|^{2}+\exp (-2 \lambda(N) t)\|\varphi\|^{2} .
$$

Выбрав $N=N(t)$ и учитьвая, что $\kappa<1 / 3$, получим неравенство (0.14) теоремы 1 при $t>2 / \lambda(2)$. 


\section{§3. О $\lambda$-последовательностях}

В этом параграфе докажем сушествование оптимальной $\bar{\lambda}$-последовательности с произвольным $\bar{x}_{0}>0$.

Лемма 2. Пусть $a, d, c-$ такие действительные числа, что $0 \leqslant a<$ $a+d \leqslant c, \Delta=(0, a) \cup(a+d, c),|\Delta|=c-d$. Тогда для любой функиии $g(x) \in C^{\infty}[0, c]$ при $2 c \geqslant 3 d$ справедливь неравенства

$$
\int_{\Delta} g^{2}(x) d x \leqslant e_{p} \int_{a}^{a+d} g^{2}(x) d x+3^{p} c^{2 p} \int_{0}^{c} g_{p}^{2}(x) d x, \quad p \geqslant 1,
$$

где $g_{p}=D_{x}^{p} g$ и числа е ер подчиняются неравенствам

$$
e_{p} \leqslant \bar{e}_{p}\left(\frac{c}{d}\right)^{2 p-1}
$$

с числами $\bar{e}_{p}$, зависящими только от $р$.

Если же $2 c \leqslant 3 d$, то выполнены неравенства

$$
\int_{\Delta} g^{2}(x) d x \leqslant \widetilde{e}_{p} \int_{a}^{a+d} g^{2}(x) d x+(4|\Delta| c)^{p} \int_{0}^{c} g_{p}^{2}(x) d x, \quad p \geqslant 1,
$$

с числами $\widetilde{e}_{p}$, зависящими только от р.

ДоКАЗАТЕЛЬСТво. Возведя равенство

$$
g(x)=g(y)+\int_{y}^{x} g^{\prime}(t) d t, \quad 0 \leqslant y<x \leqslant c,
$$

в квадрат, получаем

$$
g^{2}(x) \leqslant 2 g^{2}(y)+2 c \int_{0}^{c} g^{\prime 2}(t) d t .
$$

После интегрирования по $x \in \Delta$ и $y \in[a, a+d]$ будем иметь

$$
d \int_{\Delta} g^{2}(x) d x \leqslant 2|\Delta|\left(\int_{a}^{a+d} g^{2}(y) d y+d c \int_{0}^{c} g^{2}(t) d t\right) .
$$

Отсюда получаем начальный шаг индукции как для (3.1), так и для (3.3). Предположим, что эти неравенства справедливы до некоторого значения $p$. Установим их для значения $p+1$.

1) Пусть $2|\Delta| \geqslant d$. Заменив в (3.4) функцию $g$ на $g_{p}$ и умножив на $3^{p} c^{2 p} / d$, будем иметь

$$
3^{p} c^{2 p} \int_{\Delta} g_{p}^{2}(x) d x \leqslant \frac{2|\Delta|}{d} 3^{p} c^{2 p} \int_{a}^{a+d} g_{p}^{2}(x) d x+2 \cdot 3^{p} c^{2 p+2} \int_{0}^{c} g_{p+1}^{2}(x) d x .
$$

Сложив это неравенство с (3.1), получим при $2|\Delta| \geqslant d$

$$
\begin{aligned}
\int_{\Delta} g^{2}(x) d x \leqslant & e_{p} \int_{a}^{a+d} g^{2}(x) d x+\frac{4|\Delta|}{d} 3^{p} c^{2 p} \int_{a}^{a+d} g_{p}^{2}(x) d x \\
& +2 \cdot 3^{p} c^{2 p+2} \int_{0}^{c} g_{p+1}^{2}(x) d x .
\end{aligned}
$$


К среднему интегралу применим (1.3) с $\varepsilon=d^{2}$ и $i=p+1$. Тогда, поскольку $4|\Delta| d=4(c-d) d \leqslant c^{2}$,

$$
\begin{aligned}
\int_{\Delta} g^{2}(x) d x \leqslant & \left\{e_{p}+4 \cdot 3^{p} G_{p+1}\left(\frac{c}{d}\right)^{2 p+1}\right\} \\
& \times \int_{a}^{a+d} g^{2}(x) d x+3^{p+1} c^{2 p+2} \int_{0}^{c} g_{p+1}^{2}(x) d x .
\end{aligned}
$$

Тем самым, неравенство (3.1) доказано. Неравенство (3.2) следует из рекуррентной формулы

$$
e_{p+1}=e_{p}+4 \cdot 3^{p} G_{p+1}\left(\frac{c}{d}\right)^{2 p+1} .
$$

2) Пусть теперь $2|\Delta| \leqslant d$. Пользуясь (3.4), запишем неравенство

$$
\begin{aligned}
4^{p}(|\Delta| c)^{p} \int_{\Delta} g_{p}^{2}(x) d x \leqslant & \frac{2|\Delta|}{d} 4^{p}(|\Delta| c)^{p} \int_{a}^{a+d} g_{p}^{2}(x) d x \\
& +2 \cdot 4^{p}(|\Delta| c)^{p+1} \int_{0}^{c} g_{p+1}^{2}(x) d x .
\end{aligned}
$$

Сложив это неравенство с (3.3), получим при $2|\Delta| \leqslant d$

$$
\begin{aligned}
\int_{\Delta} g^{2}(x) d x \leqslant & \widetilde{e}_{p} \int_{a}^{a+d} g^{2}(x) d x+2 \cdot 4^{p}(|\Delta| c)^{p} \int_{a}^{a+d} g_{p}^{2}(x) d x \\
& +2 \cdot 4^{p}(|\Delta| c)^{p+1} \int_{0}^{c} g_{p+1}^{2}(x) d x
\end{aligned}
$$

$\mathrm{K}$ среднему интегралу применим (1.3) с $\varepsilon=|\Delta| c$ и $i=p+1$. Тогда неравенство

$$
\int_{\Delta} g^{2}(x) d x \leqslant\left\{\widetilde{e}_{p}+2 \cdot 4^{p} G_{p+1}\right\} \int_{a}^{a+d} g^{2}(x) d x+(4|\Delta| c)^{p+1} \int_{0}^{c} g_{p+1}^{2}(x) d x
$$

доказывает (3.3).

УТВЕРЖДЕНИЕ 2. Пусть $i=q$ или $k$ и числа $0<z \leqslant a<b \leqslant c$ удовлетворяют неравенству $2(c-z) \geqslant 3(b-a)$. Тогда

$$
\lambda^{-1}(z, c) \leqslant\left(e_{i}+1\right) \lambda^{-1}(a, b)+\frac{3^{i}}{\widehat{a}}(c-z)^{2 i} .
$$

Если, дополнительно,

$$
\widehat{a}\left(\bar{e}_{i}+1\right)(b-a)^{1-2 i} \leqslant 3^{i}(c-z) \lambda(a, b),
$$

mo

$$
\widehat{a} \leqslant 2 \cdot 3^{i}(c-z)^{2 i} \lambda(z, c)
$$


ДокАЗАТЕЛЬСтво. Применим неравенство $(3.1)$ к интервалам $(a, b) \subset(z, c)$ и функции $g(x, \mathbf{y}) \in C_{0}^{\infty}(\Omega)$, продолженной нулем вне $\Omega$, в следующем виде:

$$
\int_{\Delta} g^{2}(x, \mathbf{y}) d x \leqslant e_{i} \int_{a}^{b} g^{2}(x, \mathbf{y}) d x+3^{i}(c-z)^{2 i} \int_{z}^{c} g_{i}^{2}(x, \mathbf{y}) d x, \quad \mathbf{y} \in \mathbb{R}_{n},
$$

где $g_{i}=D_{x}^{i} g$ и $\Delta=(z, a) \cup(b, c)$. После интегрирования по $\mathbf{y} \in \mathbb{R}_{n}$ находим, что

$$
\int_{\Omega_{z}^{c}} g^{2}(x, \mathbf{y}) d x d \mathbf{y} \leqslant\left(e_{i}+1\right) \int_{\Omega_{a}^{b}} g^{2}(x, \mathbf{y}) d x d \mathbf{y}+3^{i}(c-z)^{2 i} \int_{\Omega_{z}^{c}} g_{i}^{2}(x, \mathbf{y}) d x d \mathbf{y}
$$

Применив $(0.7),(0.4),(0.5)$, получим соотношение

$$
\int_{\Omega_{z}^{c}} g^{2}(x, \mathbf{y}) d x d \mathbf{y} \leqslant\left(e_{i}+1\right) \lambda^{-1}(a, b) \int_{\Omega_{a}^{b}} F(g) d x d \mathbf{y}+\frac{3^{i}}{\widehat{a}}(c-z)^{2 i} \int_{\Omega_{z}^{c}} F(g) d x d \mathbf{y} .
$$

Тем самыг, установлено неравенство (3.5). Отсюда, пользуясь (3.2) и (3.6), выводим (3.7).

ЗАмЕчАнИЕ. Если неравенство $\lambda\left(-\infty, x_{*}\right)>0$ вьполнено при некотором $x_{*}$, то оно выполнено при любом $x>x_{*}$. В самом деле, пользуясь (3.1), запишем для функции $g(x, \mathbf{y}) \in C_{0}^{\infty}(\Omega)$ неравенства

$$
\begin{aligned}
& \int_{\Omega_{x_{*}}^{x}} g^{2}(x, \mathbf{y}) d x d \mathbf{y} \\
& \quad \leqslant e_{q} \int_{\Omega_{2 x_{*}-x}^{x_{*}}} g^{2}(x, \mathbf{y}) d x d \mathbf{y}+6^{q}\left(x-x_{*}\right)^{2 q} \int_{\Omega_{2 x_{*}-x}^{x}} g_{q}^{2}(x, \mathbf{y}) d x d \mathbf{y} \\
& \quad \leqslant\left(\frac{e_{q}}{\lambda\left(-\infty, x_{*}\right)}+\frac{6^{q}\left(x-x_{*}\right)^{2 q}}{\widehat{a}}\right) \int_{\Omega_{-\infty}^{x}} F(g) d x d \mathbf{y},
\end{aligned}
$$

из которых следует требуемое.

СлеДСТвИЕ 1. Если область удовлетворяет условию (0.9), то ג-последовательность задачи (0.1), (0.2) существует при произвольном $x_{0}>0$ с иислом $\theta \geqslant 2 \cdot 3^{q} / \widehat{a}$.

ДоКАЗАТЕЛЬСТво. Пусть уже построен элемент $\lambda$-последовательности $x_{j}$ с числом $\theta \geqslant 2 \cdot 3^{q} / \widehat{a}$ и по предположению $(0.9) \lambda\left(x_{j}, x_{*}\right) \neq 0$. В качестве следуюшего элемента $\lambda$-последовательности можно взять произвольное $x_{j+1}$, удовлетворяюшее неравенствам $x_{j+1} \geqslant x_{j}+1,2 x_{j+1} \geqslant 3 x_{*}-x_{j}, \widehat{a}\left(\bar{e}_{q}+1\right) \times$ $\left(x_{*}-x_{j}\right)^{1-2 q} \leqslant 3^{q}\left(x_{j+1}-x_{j}\right) \lambda\left(x_{j}, x_{*}\right)$. Соотношение (0.8) следует из утверждения 2 при $i=q, a=z=x_{j}, b=x_{*}, c=x_{j+1}$.

Построим специальную $\bar{\lambda}$-последовательность с $\bar{\theta} \geqslant 2 \cdot 3^{k} / \widehat{a}$ и докажем, что она является оптимальной.

Сначала докажем неравенство

$$
\varlimsup_{r \rightarrow r_{2}} \lambda\left(r_{1}, r\right) \leqslant \lambda\left(r_{1}, r_{2}\right)
$$


Выберем произвольное $\varepsilon>0$ и ненулевую функцию $f \in C_{0}^{\infty}(\Omega)$ такую, что

$$
\left(\lambda\left(r_{1}, r_{2}\right)+\varepsilon\right) \int_{\Omega_{r_{1}}^{r_{2}}} f^{2}(x, \mathbf{y}) d x d \mathbf{y} \geqslant \int_{\Omega_{r_{1}}^{r_{2}}} F(f) d x d \mathbf{y}
$$

Пользуясь абсолютной непрерывностью интеграла Лебега, нетрудно установить сушествование такого $\delta>0$, что

$$
\left(\lambda\left(r_{1}, r_{2}\right)+2 \varepsilon\right) \int_{\Omega_{r_{1}}^{r}} f^{2}(x, \mathbf{y}) d x d \mathbf{y} \geqslant \int_{\Omega_{r_{1}}^{r}} F(f) d x d \mathbf{y}
$$

при всех $r$ таких, что $\left|r-r_{2}\right|<\delta$. Отсюда следует неравенство

$$
\lambda\left(r_{1}, r\right) \leqslant \lambda\left(r_{1}, r_{2}\right)+2 \varepsilon, \quad r:\left|r-r_{2}\right|<\delta .
$$

Тем самым, (3.8) установлено.

Опишем индуктивное построение элементов $\bar{\lambda}$-последовательности. Пусть построен элемент $\bar{x}_{\nu}$. Положим

$$
\bar{x}_{\nu+1}=\inf \left\{x>\bar{x}_{\nu}: 1 \leqslant \bar{\theta} \lambda\left(\bar{x}_{\nu}, x\right)\left(x-\bar{x}_{\nu}\right)^{2[k, q]}\right\} .
$$

Непустота множества под знаком inf при условии $(0.9)$ и $\bar{\theta} \geqslant 2 \cdot 3^{k} / \widehat{a}$ вытекает из следствия 1. Благодаря (3.8) будем иметь неравенство

$$
1 \leqslant \bar{\theta} \lambda\left(\bar{x}_{\nu}, \bar{x}_{\nu+1}\right)\left(\bar{x}_{\nu+1}-\bar{x}_{\nu}\right)^{2[k, q]} .
$$

Кроме того, при любом $c \in\left(\bar{x}_{\nu}, \bar{x}_{\nu+1}\right)$ вьполнено противоположное неравенство

$$
1>\bar{\theta} \lambda\left(\bar{x}_{\nu}, c\right)\left(c-\bar{x}_{\nu}\right)^{2[k, q]}
$$

УТВЕРЖДЕНИЕ 3. Пусть $\left\{x_{j}\right\}_{j=0}^{\infty}-$ произвольная $\lambda$-последовательность. Пусть $\left\{\bar{x}_{\nu}\right\}_{\nu=0}^{\infty}-$ специальная $\bar{\lambda}$-последовательность. Тогда найдется число $D(\Xi)>1$ такое, что справедлива импликация

$$
\left(x_{L} \leqslant \bar{x}_{N}\right) \quad \Rightarrow \quad(L \leqslant(D+1) N) .
$$

ДоКАЗАТЕЛЬСтво. Достаточно установить неравенство

$$
\bar{x}_{\nu+1}-\bar{x}_{\nu} \leqslant D\left(x_{j+1}-x_{j}\right)
$$

для вложенных отрезков $\left[x_{j}, x_{j+1}\right] \subset\left[\bar{x}_{\nu}, \bar{x}_{\nu+1}\right)$. Действительно, на промежутке $\left[\bar{x}_{\nu}, \bar{x}_{\nu+1}\right)$ расположится не более $D$ целых отрезков $\left[x_{j}, x_{j+1}\right]$ и, возможно, два нецелых отрезка на конщах. Всего отрезок $\left[\bar{x}_{0}, \bar{x}_{N}\right]$ будет содержать не более $(D+1) N$ отрезков $\left[x_{j}, x_{j+1}\right]$, поэтому будет справедливо (3.11). Для доказательства (3.12) установим неравенство

$$
\left(c-\bar{x}_{\nu}\right) \leqslant D\left(x_{j+1}-x_{j}\right)
$$

при некотором $c \geqslant \bar{x}_{\nu+1}$. 
Напомним, что $\bar{\theta} \geqslant 2 \cdot 3^{k} / \widehat{a}$. Применим утверждение 2 к числам $z=\bar{x}_{\nu}, a=x_{j}$, $b=x_{j+1}$, число $c$ выберем ниже. Положим

$$
D=\max \left(\frac{3}{2}, \theta \frac{\widehat{a}\left(\bar{e}_{k}+1\right)}{3^{k}}, \theta \frac{\widehat{a}\left(\bar{e}_{q}+1\right)}{3^{q}}\right),
$$

число $c_{1}$ выберем из равенства

$$
\frac{c_{1}-\bar{x}_{\nu}}{x_{j+1}-x_{j}}=D
$$

обеспечивающего (3.13). Если окажется, что $c_{1}-\bar{x}_{\nu}<1$, то $x_{j+1}-x_{j}<1$. В таком случае при $i=k, q$

$$
\frac{3^{i}\left(c_{1}-\bar{x}_{\nu}\right)}{\theta\left(x_{j+1}-x_{j}\right)} \geqslant \widehat{a}\left(\bar{e}_{i}+1\right) \geqslant \frac{\widehat{a}\left(\bar{e}_{i}+1\right)}{\theta \lambda\left(x_{j}, x_{j+1}\right)\left(x_{j+1}-x_{j}\right)^{2 i}}
$$

и условие (3.6) выполнено. Положим $c=\max \left(c_{1}, x_{j+1}\right)$. Тогда из (3.7) при $i=k, q$ получаем, что точка $\bar{x}_{\nu+1}$ специальной $\bar{\lambda}$-последовательности должна удовлетворять неравенству $\bar{x}_{\nu+1} \leqslant c$. Поскольку $x_{j+1}<\bar{x}_{\nu+1}$, то $c=c_{1}$ и (3.13) справедливо.

Если же окажется, что $c_{1}-\bar{x}_{\nu} \geqslant 1$, то из (3.14) следует, что $D\left(x_{j+1}-x_{j}\right) \geqslant 1$. Положим $i=q$ и выберем $c_{2}$ из (3.6), считая его равенством. При этом

$$
\frac{c_{2}-\bar{x}_{\nu}}{x_{j+1}-x_{j}}=\frac{\widehat{a}\left(\bar{e}_{q}+1\right)}{3^{q} \lambda\left(x_{j}, x_{j+1}\right)\left(x_{j+1}-x_{j}\right)^{2 q}} \leqslant \theta \frac{\widehat{a}\left(\bar{e}_{q}+1\right)}{3^{q}} .
$$

Положим

$$
c=\max \left(c_{2}, \bar{x}_{\nu}+1, \bar{x}_{\nu}+\frac{3\left(x_{j+1}-x_{j}\right)}{2}, x_{j+1}\right) .
$$

Тогда при $c>x_{j+1}$ соотношения $(3.6),(3.13)$ остаются выполненными. Применяя утверждение 2 с $i=q$, из (3.7), как и раншше, устанавливаем, что $\bar{x}_{\nu+1} \leqslant c$. Утверждение 3 доказано.

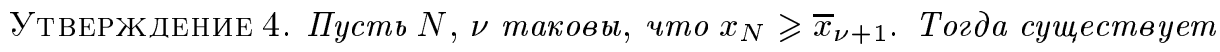
постоянная $E(\Xi) \geqslant 1$ такая, что справедливо неравенство

$$
\lambda(N) \leqslant E \lambda\left(\bar{x}_{\nu}, \bar{x}_{\nu+1}\right) .
$$

ДокаЗАтельство. Рассмотрим сначала случай, когда $\bar{x}_{\nu+1}-\bar{x}_{\nu}>1$. Тогда в силу (3.9) можно подобрать $c<\bar{x}_{\nu+1}$ так, чтобы выполнялись неравенства $\delta_{\nu}=$ $c-\bar{x}_{\nu}>1$ и

$$
\frac{1}{2} \leqslant \bar{\theta} \lambda\left(\bar{x}_{\nu}, \bar{x}_{\nu+1}\right) \delta_{\nu}^{2 q}
$$

Кроме того, ввиду (3.8) найдется такая функция $f \in C_{0}^{\infty}(\Omega), \int_{Q} f^{2} d x d y=1$, $Q=\Omega \frac{c}{x_{\nu}}$, что

$$
\int_{Q} F(f) d x d \mathbf{y} \leqslant 2 \lambda\left(\bar{x}_{\nu}, c\right) \leqslant 3 \lambda\left(\bar{x}_{\nu}, \bar{x}_{\nu+1}\right) .
$$


Покажем, что существует $\varepsilon(\Xi)>0$ такое, что

$$
\delta=\int_{\Omega_{a}^{b}} f^{2} d x d \mathbf{y} \geqslant \frac{1}{2 \widetilde{e}_{q}}
$$

где $a=\bar{x}_{\nu}+\varepsilon \delta_{\nu}, b=c-\varepsilon \delta_{\nu}$. Для этого запишем неравенство, следуюшее из (3.3):

$$
\int_{Q \backslash \Omega_{a}^{b}} f^{2} d x d \mathbf{y} \leqslant \widetilde{e}_{q} \int_{\Omega_{a}^{b}} f^{2} d x d \mathbf{y}+\frac{\left(8 \varepsilon \delta_{\nu}^{2}\right)^{q}}{\widehat{a}} \int_{Q} F(f) d x d \mathbf{y}, \quad \varepsilon \leqslant \frac{1}{6} .
$$

Из неравенства (3.19) при помоши (3.17), (3.10) выводим соотношение $1-\delta \leqslant \widetilde{e}_{q} \delta+$ $\frac{2(8 \varepsilon)^{q}}{\widehat{a} \bar{\theta}}$. Тогда при $\delta<\frac{1}{4}, \varepsilon=\min \left(\frac{1}{6}, \frac{1}{8}\left(\frac{\widehat{a} \bar{\theta}}{8}\right)^{1 / q}\right)$, поскольку $\widetilde{e}_{q}>2$, из него выводим $\delta \geqslant \frac{1}{2 \widetilde{e}_{q}}$. Тем самым, (3.18) доказано.

Теперь выберем срезаюшую функцию $h(x) \in C_{0}^{\infty}\left(\bar{x}_{\nu}, c\right)$, равную 1 на $[a, b]$, такую, что

$$
\left|D_{x}^{i} h\right| \leqslant \frac{\bar{a}_{i}}{\left(\varepsilon \delta_{\nu}\right)^{i}}, \quad \bar{a}_{i+1} \geqslant \bar{a}_{i}
$$

Тогда

$$
\begin{aligned}
I & =\int_{Q} F(h f) d x d \mathbf{y}=\int_{Q}\left(h^{2} B(f)+\sum_{i=q}^{k} a_{i}(x, \mathbf{y})\left[\sum_{p=0}^{i} C_{i}^{p} D_{x}^{p} h D_{x}^{i-p} f\right]^{2}\right) d x d \mathbf{y} \\
& \leqslant \int_{Q}\left(B(f)+\bar{a} 2^{2 k} \bar{a}_{k}^{2} \sum_{i=q}^{k} \sum_{p=0}^{i} \frac{\left(D_{x}^{i-p} f\right)^{2}}{\left(\varepsilon \delta_{\nu}\right)^{2 p}}\right) d x d \mathbf{y} .
\end{aligned}
$$

Пользуясь леммой 1 , находим, что

$$
\begin{aligned}
I & \leqslant \int_{Q}\left(B(f)+\bar{a} 2^{2 k} \bar{a}_{k}^{2} \sum_{i=q}^{k} i\left[\left(D_{x}^{i} f\right)^{2}+G_{i} \frac{f^{2}}{\left(\varepsilon \delta_{\nu}\right)^{2 i}}\right]\right) d x d \mathbf{y} \\
& \leqslant \int_{Q} B(f) d x d \mathbf{y}+\bar{a} 2^{2 k} \bar{a}_{k}^{2} k \int_{Q}\left(\frac{A(f)}{\widehat{a}}+\frac{(k-q+1) \max _{i=\overline{q, k}} G_{i} f^{2}}{\varepsilon^{2 k} \delta_{\nu}^{2 q}}\right) d x d \mathbf{y} \\
& \leqslant E_{1}(\Xi) \int_{Q}\left(F(f)+\frac{f^{2}}{\varepsilon^{2 k} \delta_{\nu}^{2 q}}\right) d x d \mathbf{y} .
\end{aligned}
$$

Далее, применяя (3.17) и (3.16), будем иметь

$$
I \leqslant E_{1}(\Xi)\left(3 \lambda\left(\bar{x}_{\nu}, \bar{x}_{\nu+1}\right)+\frac{2 \bar{\theta} \lambda\left(\bar{x}_{\nu}, \bar{x}_{\nu+1}\right)}{\varepsilon^{2 k}}\right) \leqslant E_{2}(\Xi) \lambda\left(\bar{x}_{\nu}, \bar{x}_{\nu+1}\right) .
$$

Учитывая, что

$$
\lambda(N) \leqslant I\left(\int_{Q}(h f)^{2} d x d \mathbf{y}\right)^{-1} \leqslant I\left(\int_{\Omega_{a}^{b}} f^{2} d x d \mathbf{y}\right)^{-1} \leqslant 2 \widetilde{e}_{q} E_{2}(\Xi) \lambda\left(\bar{x}_{\nu}, \bar{x}_{\nu+1}\right),
$$

устанавливаем неравенство (3.15).

Доказательство утверждения 4 в случае $\bar{x}_{\nu+1}-\bar{x}_{\nu} \leqslant 1$ проводится аналогично. 
СлЕДСТВИЕ 2. При $t>\max \left\{\bar{\lambda}^{-1}(1), \lambda^{-1}(1)\right\}$ справедливо неравенство

$$
N(t) \leqslant C(\Xi) \bar{N}(t), \quad C(\Xi)=2(D+E) .
$$

ДокаЗАТЕЛЬСТво. Пусть $N(t)>(D+1)(\bar{N}(t)+1)$. По утверждению 3 имеем $x_{N(t)} \geqslant \bar{x}_{\bar{N}(t)+1}$. По утверждению 4 , примененному ко всем $\nu \leqslant \bar{N}(t)$, имеем $\lambda(N(t)) \leqslant E \lambda\left(\bar{x}_{\nu}, \bar{x}_{\nu+1}\right)$. Кроме того, справедливы неравенства $\lambda(N(t)) \leqslant \lambda(1) \leqslant$ $\lambda\left(-\infty, x_{0}\right)=\lambda\left(-\infty, \bar{x}_{0}\right)$. Поэтому имеем неравенство $\lambda(N(t)) \leqslant E \bar{\lambda}(\bar{N}(t)+1)$. Отсюда $N(t) \leqslant t \lambda(N(t)) \leqslant E t \bar{\lambda}(\bar{N}(t)+1) \leqslant E(\bar{N}(t)+1)$. Следствие доказано.

\section{§4. Примеры}

Для областей типа слоя (0.16) естественно выразить оценку (0.14) в терминах функции $f(x)$. С этой целью докажем следующее утверждение.

Лемма 3. Рассмотрим область $Q=\left\{(x, y) \in \mathbb{R}_{2}: 0<x<r, y>0\right\} u$ функцию $g(x, y) \in C_{0}^{\infty}\left(\mathbb{R}_{2}\right)$, равную нулю в окрестности луча $\left\{(x, y) \in \mathbb{R}_{2}:\right.$ $x=0, y>h\}$. Справедливо обобщенное неравенство Фридрихса-Стеклова

$$
\int_{Q} g^{2}(x, y) d x d y \leqslant E_{p}\left\{r^{2 i} \int_{Q}\left(D_{x}^{i} g\right)^{2} d x d y+h^{2 p} \int_{Q}\left(D_{y}^{p} g\right)^{2} d x d y\right\}, \quad p, i \in \mathbb{N},
$$

$E_{p}$ - положительная постоянная, зависящая от $p$.

ДоКАЗАТЕЛЬСтво. Ввиду того что $g(0, y)=0$ при $y>h$, можем записать неравенство Фридрихса-Стеклова при каждом фиксированном $y, y>h$ :

$$
\int_{0}^{r} g^{2}(x, y) d x \leqslant r^{2} \int_{0}^{r}\left(D_{x} g\right)^{2}(x, y) d x
$$

Применяя последнее $i$ раз и интегрируя по $y, y>h$, устанавливаем, что

$$
\int_{0}^{r} \int_{y>h} g^{2}(x, y) d x d y \leqslant r^{2 i} \int_{0}^{r} \int_{y>h}\left(D_{x}^{i} g\right)^{2}(x, y) d x d y .
$$

Далее, при каждом $x, 0<x<r$, запишем неравенство

$$
\int_{0}^{h} g^{2}(x, y) d y \leqslant e_{p} \int_{h}^{2 h} g^{2}(x, y) d y+12^{p} h^{2 p} \int_{0}^{2 h}\left(D_{y}^{p} g\right)^{2}(x, y) d y,
$$

которое следует из (3.1) при $a=h, d=h, c=2 h$. Проинтегрировав последнее по $x, 0<x<r$, выводим

$$
\int_{0}^{r} \int_{0}^{h} g^{2}(x, y) d x d y \leqslant e_{p} \int_{0}^{r} \int_{h}^{2 h} g^{2} d x d y+12^{p} h^{2 p} \int_{0}^{r} \int_{0}^{2 h}\left(D_{y}^{p} g\right)^{2} d x d y .
$$

Соединяя (4.2), (4.3), получаем требуемое (4.1). 
УТВЕРЖДЕНИЕ 5. Пусть коэффициенты уравнения (0.1) подчиняются дополнительному требованию (0.15). Тогда $\Pi^{s}$-последовательность является $\lambda$-последовательностью для $\Omega[f]$.

ДоКАЗАТЕЛЬСТво выведем из леммы 3.

Функцию $g(x, \mathbf{y}) \in C_{0}^{\infty}(\Omega[f])$ продолжим нулем вне $\Omega[f]$. Обозначим через $\widehat{x}_{\boldsymbol{j}}$ точку минимума функции $f(x)$ на $\left[x_{j}, x_{j+1}\right]: f\left(\widehat{x}_{j}\right)=\min _{\left[x_{j}, x_{j+1}\right]} f(x)$. Рассмотрим область $Q=\left\{\left(x, y_{1}\right) \in \mathbb{R}_{2}: x_{j}<x<x_{j+1},\left|y_{1}\right|<f(x)\right\}$. Обозначим

$$
\begin{aligned}
& Q_{1}^{ \pm}=\left\{\left(x, y_{1}\right) \in \mathbb{R}_{2}: x_{j}<x<\widehat{x}_{j}, \pm y_{1}>0\right\}, \\
& Q_{2}^{ \pm}=\left\{\left(x, y_{1}\right) \in \mathbb{R}_{2}: \widehat{x}_{j}<x<x_{j+1}, \pm y_{1}>0\right\} .
\end{aligned}
$$

Предположим сначала, что $\Delta_{j} \geqslant 1$. Воспользовавшись неравенством (4.1) для полуполосы $Q_{1}^{+}$, получим

$$
\begin{aligned}
& \int_{Q_{1}^{+}} g^{2}(x, \mathbf{y}) d x d y_{1} \\
& \quad \leqslant E_{s}\left\{\left(\widehat{x}_{j}-x_{j}\right)^{2 q} \int_{Q_{1}^{+}}\left(D_{x}^{q} g\right)^{2} d x d y_{1}+f^{2 s}\left(\widehat{x}_{j}\right) \int_{Q_{1}^{+}}\left(D_{y_{1}}^{s} g\right)^{2} d x d y_{1}\right\}
\end{aligned}
$$

Аналогичные неравенства установим для $Q_{1}^{-}, Q_{2}^{+}, Q_{2}^{-}$. Отметим, что из определения $\Pi^{s}$-последовательности следует равенство $f^{2 s}\left(\widehat{x}_{j}\right)=\Delta_{j}^{2 q}$. Сложив четыре неравенства, будем иметь

$$
\int_{Q} g^{2}(x, \mathbf{y}) d x d y_{1} \leqslant E_{s} \Delta_{j}^{2 q} \int_{Q}\left\{\left(D_{x}^{q} g\right)^{2}+\left(D_{y_{1}}^{s} g\right)^{2}\right\} d x d y_{1}
$$

Аналогично при $\Delta_{j}<1$ установим неравенство

$$
\int_{Q} g^{2}(x, \mathbf{y}) d x d y_{1} \leqslant E_{m} \Delta_{j}^{2 k} \int_{Q}\left\{\left(D_{x}^{k} g\right)^{2}+\left(D_{y_{1}}^{m} g\right)^{2}\right\} d x d y_{1} .
$$

Таким образом, из двух неравенств имеем

$$
\begin{aligned}
& \int_{Q} g^{2}(x, \mathbf{y}) d x d y_{1} \\
& \quad \leqslant \max \left(E_{s}, E_{m}\right) \Delta_{j}^{2[k, q]} \int_{Q}\left[\left(D_{x}^{k} g\right)^{2}+\left(D_{x}^{q} g\right)^{2}+\left(D_{y_{1}}^{m} g\right)^{2}+\left(D_{y_{1}}^{s} g\right)^{2}\right] d x d y_{1} .
\end{aligned}
$$

Проинтегрируем последнее по $y^{\prime}=\left(y_{2}, y_{3}, \ldots, y_{n}\right) \in \mathbb{R}_{n-1}$ :

$$
\begin{aligned}
& \int_{\Omega_{x_{j}}^{x_{j}+1}[f]} g^{2}(x, \mathbf{y}) d x d \mathbf{y} \leqslant \max \left(E_{s}, E_{m}\right) \Delta_{j}^{2[k, q]} \\
& \quad \times \int_{\Omega_{x_{j}}^{x_{j} j+1}[f]}\left\{\left(D_{x}^{k} g\right)^{2}+\left(D_{x}^{q} g\right)^{2}+\left(D_{y_{1}}^{m} g\right)^{2}+\left(D_{y_{1}}^{s} g\right)^{2}\right\} d x d \mathbf{y} .
\end{aligned}
$$


Используя условия $(0.15),(0.4)$, получаем

$$
\int_{\Omega_{x_{j}}^{x_{j} j+1}[f]} g^{2}(x, \mathbf{y}) d x d \mathbf{y} \leqslant \frac{\max \left(E_{s}, E_{m}\right)}{\min (\widehat{a}, \widehat{b})} \Delta_{j}^{2[k, q]} \int_{\Omega_{x_{j}}^{x_{j}+1}[f]} F(g) d x d \mathbf{y} .
$$

Таким образом, имеем оценку

$$
1 \leqslant \frac{\max \left(E_{s}, E_{m}\right)}{\min (\widehat{a}, \widehat{b})} \Delta_{j}^{2[k, q]} \lambda\left(x_{j}, x_{j+1} ; \Omega[f]\right) .
$$

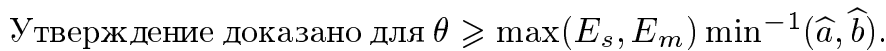

Для функции $\rho^{[a, b]}$ приведем некоторые неравенства, которые будут использованы в дальнейшем:

$$
\begin{aligned}
& \text { если } \quad \rho \leqslant \varrho, \text { то } \quad \rho^{[a, b]} \leqslant \varrho^{[a, b]} ; \\
& \text { если } c \geqslant 1, \text { то }(c \rho)^{[a, b]} \leqslant \rho^{[a, b]} c^{\max (a, b)} .
\end{aligned}
$$

Поскольку для $\Pi^{s}$-последовательности справедливы неравенства

$$
\int_{x_{j}}^{x_{j+1}} \frac{d x}{f^{[m / k, s / q]}(x)} \leqslant \frac{x_{j+1}-x_{j}}{\min _{\left[x_{j}, x_{j+1}\right]} f^{[m / k, s / q]}(x)}=1, \quad j=\overline{0, \infty},
$$

TO

$$
\int_{x_{0}}^{x_{N}} \frac{d x}{f^{[m / k, s / q]}(x)} \leqslant N, \quad N \in \mathbb{N} .
$$

Обозначим $\delta(N)=\max _{j=\overline{0, N-1}} \Delta_{j}, N \in \mathbb{N}$. Поскольку $\Pi^{s}$-последовательность явлется $\lambda$-последовательностью для $\Omega[f]$, то имеем

$$
\rho_{*}^{2[k, q]}\left(x_{N}\right) \geqslant \delta^{2[k, q]}(N)=\max _{j=\overline{0, N-1}} \Delta_{j}^{2[k, q]} \geqslant \frac{1}{\theta \min _{j=\overline{0, N-1}} \lambda\left(x_{j}, x_{j+1}\right)} .
$$

Кроме того, ввиду (0.11) можно подобрать $\epsilon, 0<\epsilon<1$ :

$$
\rho_{*}^{2[k, q]}\left(x_{N}\right) \geqslant \delta^{2[k, q]}(N) \geqslant \delta^{2[k, q]}(1)=\Delta_{0}^{2[k, q]} \geqslant \frac{\epsilon}{\theta \lambda\left(-\infty, x_{0}\right)} .
$$

В итоге установлено неравенство

$$
\lambda(N) \geqslant \frac{\epsilon}{\theta \delta^{2[k, q]}(N)} \geqslant \frac{\epsilon}{\theta \rho_{*}^{2[k, q]}\left(x_{N}\right)}, \quad N \in \mathbb{N} .
$$

УТВЕРЖДЕНИЕ 6. Для областей $\Omega[f]$ вида (0.16) при условии (0.17) и дополнительном требовании (0.15) на коэффициенты уравнения решение задачи (0.1)-(0.3) при $t \geqslant T_{1}$ удовлетворяет оценке $(0.20)$ :

$$
\|u(t)\|_{\Omega[f]} \leqslant M_{1} \exp \left(-\kappa_{1} \int_{x_{0}}^{r(t)} \frac{d x}{f^{[m / k, s / q]}(x)}\right)\|\varphi\|_{\Omega[f]},
$$

в которой функция $r(t), t \geqslant 0$, определяется равенством (0.19). Здесь $\kappa_{1}\left(\Xi, R_{0}, m, s, f\right), M_{1}(\Xi), T_{1}\left(R_{0}, f, m, s, k, q\right)$ - положсительные постоянные. 
ДокАЗАТЕЛЬСтво. Пусть $\left\{x_{j}\right\}_{j=0}^{\infty}-\Pi^{s}$-последовательность, для нее справедливо неравенство (4.5). Ввиду (0.15) согласно утверждению 5 такая последовательность является $\lambda$-последовательностью для области $\Omega[f]$, поэтому имеет место неравенство (4.6).

Отметим, что из условия $(0.17)$ следует, что $\lim _{t \rightarrow \infty} r(t)=\infty$. Поэтому можем выбрать $T_{1}$ такое, что при $t \geqslant T_{1} r(t) \geqslant x_{2}$. Зафиксируем $t, t \geqslant T_{1}$. Пусть $r(t) \in\left[x_{N}, x_{N+1}\right)$. Тогда по (4.6) имеем

$$
\lambda(N) \geqslant \frac{\epsilon}{\theta \rho_{*}^{2[k, q]}\left(x_{N}\right)} \geqslant \frac{\epsilon}{\theta \rho_{*}^{2[k, q]}(r(t))} .
$$

Далее, согласно (4.5) установим

$$
\int_{x_{0}}^{r(t)} \frac{d x}{f^{[m / k, s / q]}(x)} \leqslant \int_{x_{0}}^{x_{N+1}} \frac{d x}{f^{[m / k, s / q]}(x)} \leqslant N+1 .
$$

Подставляя (4.7), (4.8) в (2.15), получаем

$$
\begin{aligned}
\|u(t)\|_{\Omega[f]}^{2} \leqslant & {\left[\widetilde{M} \exp \left(-2 \kappa\left(\int_{x_{0}}^{r(t)} \frac{d x}{f^{[m / k, s / q]}(x)}-1\right)\right)\right.} \\
& \left.+\exp \left(-\frac{2 \epsilon t}{\theta \rho_{*}^{2[k, q]}(r(t))}\right)\right]\|\varphi\|_{\Omega[f]}^{2} .
\end{aligned}
$$

Отсюда благодаря (0.19) выводим (0.20).

Конечно, в обшем случае оценка ( 0.20$)$ оказывается загрубленной по отношению к оценке (0.14). Докажем, что если вьполнено условие $(0.21)$ и $s=l$, то оценка будет того же порядка.

Заметим сначала, что если непрерывные функции $f(x), g(x)$, определенные на $\mathbb{R}$, удовлетворяют неравенству $g(x) \leqslant f(x), x \in[a, b]$, то

$$
\lambda(a, b ; \Omega[f]) \leqslant \lambda(a, b ; \Omega[g])
$$

Это следует из того, что для $\Omega[g]$ сужается множество, по которому берется инфимум в (0.7). Рассмотрим теперь плоский слой $\Omega[h], h=$ const, и установим оценки

$$
\gamma_{1} h^{-2[m, l]} \leqslant \lambda(a, b ; \Omega[h]) \leqslant \gamma_{2} h^{-2[m, l]} .
$$

Положим

$$
G(g)=\sum_{i=q}^{k}\left(D_{x}^{i} g\right)^{2}+\sum_{i=l}^{m} \sum_{|\alpha|=i}\left(D_{\mathbf{y}}^{\alpha} g\right)^{2}
$$

тогда ввиду неравенства $F(g) \leqslant C_{1} G(g)$, вытекающего из ограниченности коэффициентов уравнения $(0.1)$, имеем

$$
\lambda(a, b ; \Omega[h]) \leqslant C_{1} \inf _{g(x, \mathbf{y}) \in C_{0}^{\infty}(\Omega[h])}\left\{\int_{\Omega_{a}^{b}[h]} G(g) d x d \mathbf{y}: \int_{\Omega_{a}^{b}[h]} g^{2} d x d \mathbf{y}=1\right\} .
$$


Аналогично при $j=l$ или $m$ из (0.15), справедливого для $s=l$, следует неравенство

$\lambda(a, b ; \Omega[h]) \geqslant \widehat{b} \inf _{g(x, \mathbf{y}) \in C_{0}^{\infty}(\Omega[h])}\left\{\int_{\Omega_{a}^{b}[h]}\left(D_{y_{1}}^{j} g\right)^{2} d x d \mathbf{y}: \int_{\Omega_{a}^{b}[h]} g^{2} d x d \mathbf{y}=1\right\} \equiv \widehat{b} \lambda_{j}$.

Очевидно,

$\lambda \equiv \inf _{g(x, \mathbf{y}) \in C_{0}^{\infty}(\Omega[h])} \frac{\int_{\Omega_{a}^{b}[h]} \sum_{i=l}^{m}\left(D_{y_{1}}^{i} g\right)^{2} d x d \mathbf{y}}{\int_{\Omega_{a}^{b}[h]} g^{2} d x d \mathbf{y}} \leqslant \inf _{g(x, \mathbf{y}) \in C_{0}^{\infty}(\Omega[h])} \frac{\int_{\Omega_{a}^{b}[h]} G(g) d x d \mathbf{y}}{\int_{\Omega_{a}^{b}[h]} g^{2} d x d \mathbf{y}}$.

Легко видеть, что в действительности в (4.11) имеет место равенство. Поэтому $\widehat{b} \lambda_{j} \leqslant \lambda(a, b ; \Omega[h]) \leqslant C_{1} \lambda$. Если $h \geqslant 1$, то, совершая замену переменных $y_{1}=h \tau$, получаем

$$
\begin{aligned}
\lambda & =\inf _{g\left(y_{1}\right) \in C_{0}^{\infty}(-h, h)} \frac{\int_{-h}^{h} \sum_{i=l}^{m}\left(D_{y_{1}}^{i} g\right)^{2} d y_{1}}{\int_{-h}^{h} g^{2} d y_{1}} \\
& =\inf _{g(\tau) \in C_{0}^{\infty}(-1,1)} \frac{\int_{-1}^{1} \sum_{i=l}^{m} h^{-2 i}\left(D_{\tau}^{i} g\right)^{2} d \tau}{\int_{-1}^{1} g^{2} d \tau} \leqslant \frac{C_{2}}{h^{2 l}} .
\end{aligned}
$$

Таким образом,

$$
\frac{C_{3}}{h^{2 l}}=\widehat{b} \lambda_{l} \leqslant \lambda(a, b ; \Omega[h]) \leqslant \frac{C_{4}}{h^{2 l}} .
$$

Если же $h<1$, то совершенно аналогично, вынося в (4.12) множитель $1 / h^{2 m}$, получаем

$$
\frac{C_{3}}{h^{2 m}}=\widehat{b} \lambda_{m} \leqslant \lambda(a, b ; \Omega[h]) \leqslant \frac{C_{4}}{h^{2 m}} .
$$

Неравенства (4.10) установлены.

УТВЕРЖДЕНИЕ 7. Пусть выполнены условия (0.15), (0.21) u $s=l \leqslant m$. Тогда для областей $\Omega[f]$ вида (0.16) оценка (0.20) решения задачи (0.1)-(0.3) будет того же порядка, что и оченка (0.14). При этом $\Pi^{l}$-последовательность является оптимальной.

ДокАЗАТЕЛЬСтво. Для первого утверждения достаточно установить неравенства

$$
\begin{gathered}
\alpha_{1} N \leqslant \int_{x_{0}}^{r} \frac{d x}{f^{[m / k, l / q]}(x)} \leqslant \alpha_{2} N, \\
\beta_{1} \rho_{*}^{-2[k, q]}(r) \leqslant \lambda(N) \leqslant \beta_{2} \rho_{*}^{-2[k, q]}(r)
\end{gathered}
$$


при $r \in\left[x_{N}, x_{N+1}\right), N \geqslant 1$. Правое неравенство (4.13) и левое (4.14) уже доказаны (см. неравенства $(4.8),(4.7))$. Из определения $\Pi^{l}$-последовательности имеем

$$
\Delta_{j}=x_{j+1}-x_{j}=f^{[m / k, l / q]}\left(\widehat{x}_{j}\right),
$$

где $\min _{\left[x_{j}, x_{j+1}\right]} f(x)=f\left(\widehat{x}_{j}\right)$. Пользуясь $\left(0.18^{\prime}\right)$, из неравенства $(0.21)$ вьводим

$$
\max \left\{f(z): z \in\left[\widehat{x}_{j}-\Delta_{j}, \widehat{x}_{j}+\Delta_{j}\right]\right\} \leqslant c f\left(\widehat{x}_{j}\right) .
$$

Ввиду включения $\left[x_{j}, x_{j+1}\right] \subset\left[\widehat{x}_{j}-\Delta_{j}, \widehat{x}_{j}+\Delta_{j}\right]$ имеем

$$
f\left(\widehat{x}_{j}\right) \leqslant f(x) \leqslant c f\left(\widehat{x}_{j}\right), \quad x \in\left[x_{j}, x_{j+1}\right] .
$$

Применяя (4.4), $\left(0.18^{\prime}\right)$, устанавливаем

$$
\begin{aligned}
\int_{x_{j}}^{x_{j+1}} \frac{d x}{f^{[m / k, l / q]}(x)} & \geqslant \frac{x_{j+1}-x_{j}}{\max _{\left[x_{j}, x_{j+1}\right]} f^{[m / k, l / q]}(x)} \geqslant \frac{x_{j+1}-x_{j}}{(c f)^{[m / k, l / q]}\left(\widehat{x}_{j}\right)} \\
& \geqslant \frac{1}{c^{\max (m / k, l / q)}} .
\end{aligned}
$$

Следовательно, справедливо неравенство

$$
\int_{x_{0}}^{r} \frac{d x}{f^{[m / k, l / q]}(x)} \geqslant \int_{x_{0}}^{x_{N}} \frac{d x}{f^{[m / k, l / q]}(x)} \geqslant \frac{N}{c^{\max (m / k, l / q)}} .
$$

Итак, неравенства (4.13) доказаны.

Далее, выведем неравенства

$$
\delta(N) \leqslant \rho_{*}\left(x_{N}\right) \leqslant 2 \delta(N), \quad N \geqslant 1 .
$$

Левое неравенство очевидно, докажем правое. Пусть $z_{*}$ - вершина прямоугольника $P^{l}\left(\rho_{*}\left(x_{N}\right), z_{*}\right)$ максимальной ширины $\rho_{*}\left(x_{N}\right)$, содержашегося в $\Gamma_{x_{0}}^{x_{N}}(f)$. Cередина его стороны $-z_{*}+\rho_{*}\left(x_{N}\right) / 2$. Пусть $x_{j_{*}}$ и $x_{j_{*}+1}$ - точки на оси $O x$, ближайшие к $z_{*}+\rho_{*}\left(x_{N}\right) / 2$ слева и справа соответственно. Если $z_{*}-x_{j_{*}} \geqslant 0$ или $x_{j_{*}+1}-$ $z_{*} \geqslant \rho_{*}\left(x_{N}\right)$, то $x_{j_{*}+1}-x_{j_{*}} \geqslant \rho_{*}\left(x_{N}\right) / 2$. Иначе $z_{*}<x_{j_{*}}<x_{j_{*}+1}<z_{*}+\rho_{*}\left(x_{N}\right)$. Тогда прямоугольник $P^{l}\left(\Delta_{j_{*}}, x_{j_{*}}\right) \subset P^{l}\left(\rho_{*}\left(x_{N}\right), z_{*}\right)$, поэтому не является максимальным, каковым его выбирали при построении $x_{j_{*}+1}$. Получили противоречие. Итак, справедливо правое неравенство (4.15).

Докажем неравенства

$$
\gamma_{3} f^{-2[m, l]}\left(\widehat{x}_{j}\right) \leqslant \lambda(a, b ; \Omega[f]) \leqslant \gamma_{2} f^{-2[m, l]}\left(\widehat{x}_{j}\right),
$$

справедливые для любого отрезка $[a, b] \subset\left[x_{j}, x_{j+1}\right]$. Они следуют из (4.9) благодаря $\left(0.21^{\prime}\right)$ и $(4.10)$.

Пользуясь равенством $\left(0.18^{\prime}\right)$, из $(4.16),(4.6)$ нетрудно вывести неравенства

$$
\gamma_{4} \delta^{-2[k, q]}(N) \leqslant \lambda(N) \leqslant \gamma_{2} \delta^{-2[k, q]}(N), \quad N \geqslant 1
$$


Очевидно, $f\left(\widehat{x}_{j+1}\right) \leqslant f\left(x_{j+1}\right)$, поэтому из $\left(0.21^{\prime}\right)$ имеем $f\left(\widehat{x}_{j+1}\right) \leqslant c f\left(\widehat{x}_{j}\right)$. Легко видеть, что верно и симметричное неравенство $f\left(\widehat{x}_{j}\right) \leqslant c f\left(\widehat{x}_{j+1}\right)$. Снова, используя равенства $\left(0.18^{\prime}\right)$, при помощи (4.4) оцениваем отношения

$$
\frac{\Delta_{j+1}}{\Delta_{j}} \leqslant c^{\max (m / k, l / q)}, \quad j=\overline{0, \infty}
$$

Следовательно,

$$
\begin{aligned}
\delta(N+1) & =\max \left\{\delta(N), \Delta_{N}\right\} \leqslant \max \left\{\delta(N), c^{\max (m / k, l / q)} \delta(N)\right\} \\
& \leqslant c^{\max (m / k, l / q)} \delta(N), \quad N \geqslant 1 .
\end{aligned}
$$

Далее, из (4.17), (4.19), (4.15) выводим

$$
\lambda(N) \leqslant \frac{\gamma_{2}}{\delta^{2[k, q]}(N)} \leqslant \frac{\gamma_{5}}{\delta^{2[k, q]}(N+1)} \leqslant \frac{\beta_{2}}{\rho_{*}^{2[k, q]}\left(x_{N+1}\right)} \leqslant \frac{\beta_{2}}{\rho_{*}^{2[k, q]}(r)} .
$$

Неравенства (4.14) установлены. Первая часть утверждения доказана.

При доказательстве второй части утверждения величины, относящиеся к $\Pi^{l}$-последовательности $\left\{\bar{x}_{j}\right\}_{j=0}^{\infty}$, будем помечать чертой сверху. Сначала установим для $\Pi^{l}$-последовательности импликацию утверждения 3 . Пусть $\left\{x_{j}\right\}_{j=0}^{\infty}-$ произвольная $\lambda$-последовательность. Для вложенных отрезков $\left[x_{j}, x_{j+1}\right] \subset\left[\bar{x}_{\nu}, \bar{x}_{\nu+1}\right)$ из определения $\lambda$-последовательности, $(4.16)$ и $\left(0.18^{\prime}\right)$ следуют неравенства

$$
\Delta_{j}^{2[k, q]} \geqslant \frac{1}{\theta \lambda\left(x_{j}, x_{j+1}\right)} \geqslant \frac{f^{2[m, l]}\left(\widehat{\bar{x}}_{\nu}\right)}{\theta \gamma_{2}}=\frac{\bar{\Delta}_{\nu}^{2[k, q]}}{\theta \gamma_{2}},
$$

доказывающие импликацию.

Докажем неравенство (3.15) утверждения 4. Обозначим $Q_{\nu}=P^{l}\left(\bar{\Delta}_{\nu}, \bar{x}_{\nu}\right) \times$ $\left\{y^{\prime}=\left(y_{2}, y_{3}, \ldots, y_{n}\right) \in \mathbb{R}_{n-1}\right\}$. При $x_{N} \geqslant \bar{x}_{\nu+1}$ ввиду вложений $Q_{\nu} \subset \Omega_{\bar{x}_{\nu}}+1[f] \subset$ $\Omega^{x_{N}}[f] \subset \Omega[f]$ из $(0.12)$ следует

$$
\lambda(N) \leqslant C_{1} \inf _{g(x, \mathbf{y}) \in C_{0}^{\infty}\left(Q_{\nu}\right)}\left\{\int_{Q_{\nu}} G(g) d x d \mathbf{y}: \int_{Q_{\nu}} g^{2} d x d \mathbf{y}=1\right\} .
$$

Далее,

$$
\begin{aligned}
& \lambda_{*} \equiv \inf _{g(x, \mathbf{y}) \in C_{0}^{\infty}\left(Q_{\nu}\right)} \frac{\int_{Q_{\nu}}\left\{\sum_{i=q}^{k}\left(D_{x}^{i} g\right)^{2}+\sum_{i=l}^{m}\left(D_{y_{1}}^{i} g\right)^{2}\right\} d x d \mathbf{y}}{\int_{Q_{\nu}} g^{2} d x d \mathbf{y}} \\
& \leqslant \inf _{g(x, \mathbf{y}) \in C_{0}^{\infty}\left(Q_{\nu}\right)} \frac{\int_{Q_{\nu}} G(g) d x d \mathbf{y}}{\int_{Q_{\nu}} g^{2} d x d \mathbf{y}}
\end{aligned}
$$


Легко видеть, что в действительности в (4.21) имеет место равенство. Поэтому $\lambda(N) \leqslant C_{1} \lambda_{*}$. Если $\bar{\Delta}_{\nu} \geqslant 1$, то, совершая замену переменных $x=\bar{\Delta}_{\nu} \sigma+\bar{x}_{\nu}$, $y_{1}=\bar{\Delta}_{\nu}^{q / l} \tau$, получаем

$$
\begin{aligned}
\lambda_{*} & =\inf _{g\left(x, y_{1}\right) \in C_{0}^{\infty}\left(P^{l}\left(\bar{\Delta}_{\nu}, \bar{x}_{\nu}\right)\right)} \frac{\int_{P^{l}\left(\bar{\Delta}_{\nu}, \bar{x}_{\nu}\right)}\left\{\sum_{i=q}^{k}\left(D_{x}^{i} g\right)^{2}+\sum_{i=l}^{m}\left(D_{y_{1}}^{i} g\right)^{2}\right\} d x d y_{1}}{\int_{P^{l}\left(\bar{\Delta}_{\nu}, \bar{x}_{\nu}\right)} g^{2} d x d y_{1}} \\
& =\inf _{g(\sigma, \tau) \in C_{0}^{\infty}\left(P^{l}(1,0)\right)} \frac{\int_{0}^{1} \int_{0}^{1}\left\{\sum_{i=q}^{k} \bar{\Delta}_{\nu}^{-2 i}\left(D_{\sigma}^{i} g\right)^{2}+\sum_{i=l}^{m} \bar{\Delta}_{\nu}^{-2 q i / l}\left(D_{\tau}^{i} g\right)^{2}\right\} d \sigma d \tau}{\int_{0}^{1} \int_{0}^{1} g^{2} d \sigma d \tau} \\
& \leqslant \frac{C_{2}}{\bar{\Delta}_{\nu}^{2 q}} .
\end{aligned}
$$

Таким образом,

$$
\lambda(N) \leqslant \frac{C_{3}}{\bar{\Delta}_{\nu}^{2 q}}
$$

и неравенство (0.8), записанное для П-последовательности $\left\{\bar{x}_{j}\right\}_{j=0}^{\infty}$, обеспечивает (3.15). Если же $\bar{\Delta}_{\nu}<1$, то рассуждаем аналогично, при этом в (4.22) делаем замену переменных $x=\bar{\Delta}_{\nu} \sigma+\bar{x}_{\nu}, y_{1}=\bar{\Delta}_{\nu}^{k / m} \tau$ и выносим множитель $C_{2} / \bar{\Delta}_{\nu}^{2 k}$.

Далее, оптимальность $\Pi^{l}$-последовательности устанавливается точно так же, как в следствии 2. Утверждение 7 доказано.

Для монотонно неубывающей функции, удовлетворяющей (0.21), справедливо неравенство

$$
\varepsilon_{1} f^{[m / k, s / q]}(r) \leqslant \rho_{*}(r) \leqslant f^{[m / k, s / q]}(r), \quad r>0 .
$$

В самом деле, пусть $P^{s}\left(\rho_{*}, z_{*}\right)$ - прямоугольник максимальной ширины $\rho_{*}$, содержащийся в $\Gamma_{x_{0}}^{r}(f)$. Ввиду монотонного неубывания функции $f(x)$ справедливо равенство $f^{[m / k, s / q]}\left(z_{*}\right)=r-z_{*}=\rho_{*}(r)$. Согласно $(0.21)$ имеем

$$
f(r)=\max _{\left[z_{*}-f^{[m / k, s / q]}\left(z_{*}\right), z_{*}+f^{[m / k, s / q]}\left(z_{*}\right)\right]} f(z) \leqslant c f\left(z_{*}\right),
$$

откуда следует правое неравенство $(4.23) \mathrm{c} \varepsilon_{1}=c^{-\max (m / k, s / q)}$.

Тогда функция $r(t), t \geqslant 0$, в неравенстве $(0.20)$ может быть определена из равенства

$$
\int_{x_{0}}^{r} \frac{d x}{f^{[m / k, s / q]}(x)}=\frac{t}{f^{2[m, s]}(r)} .
$$

В частности, взяв функцию $f(r)=r^{a}, a<q / s, r>0$, можно определить функцию

$$
r(t)=t^{q /(q-s a+2 q s a)}, \quad t \geqslant 0 .
$$

Тогда оценка (0.20) приобретает вид (0.22). При $k=q=m=l=2$ будем иметь

$$
\|u(t)\|_{\Omega[f]} \leqslant M_{2} \exp \left(-\kappa_{2} t^{(1-a) /(1+3 a)}\right)\|\varphi\|_{\Omega[f]} .
$$

При несколько других краевых условиях для уравнений 4-го порядка в работе [10] была получена такая же по форме оценка и установлена ее точность. 


\section{$\S 5$. Оценка снизу}

Доказательство теоремы 2 основано на неравенстве Гарнака, установленном Ю. Мозером в [14]. Сформулируем неравенство Гарнака в удобном для нас виде: для неотрицательного в цилиндре $Q=\left(0,9 \rho^{2}\right] \times B(2 \rho, \mathbf{0}) \subset \mathbb{R}_{n+1}$ решения уравнения (0.1) при $k=q=m=l=1$ справедливо неравенство

$$
\max _{Q^{-}} u(t, x, \mathbf{y}) \leqslant \widehat{H} \min _{Q^{+}} u(t, x, \mathbf{y}),
$$

в котором постоянная $\widehat{H} \geqslant 1$ зависит только от $n$ и констант параболичности уравнения второго порядка,

$$
\begin{aligned}
& Q^{-}=\left[\rho^{2}, 2 \rho^{2}\right] \times B(\rho, \mathbf{w}), \\
& Q^{+}=\left[8 \rho^{2}, 9 \rho^{2}\right] \times B(\rho, \mathbf{w}) .
\end{aligned}
$$

Из неравенства Гарнака вытекает следующее утверждение.

ЛЕмма 4. Пусть $\widehat{M}=M \cup B(2 \rho ;(0, \mathbf{0})) \cup B(2 \rho ;(1, \mathbf{0}))$, әде $M-$ модельная трубка из введения, $\rho=a / 8$. Тогда найдутся числа $h>16 \rho^{2}=a^{2} / 4, H>0$ такие, что неотрицательное в $\widehat{D}=(0, \infty) \times \widehat{M}$ решение $и(t, x, \mathbf{y})$ уравнения (0.1) при $k=q=m=l=1$ удовлетворяет неравенству

$$
u\left(\rho^{2}, 0, \mathbf{0}\right) \leqslant H u\left(\rho^{2}+h, 1, \mathbf{0}\right) .
$$

ДокАЗАТЕЛЬСТво. В силу условия леммы цилиндр $Q$ лежит в $\widehat{D}$. Положим $t_{0}=\rho^{2}, t_{1}=8 \rho^{2}+t_{0}, \mathbf{v}_{0}=(0, \mathbf{0}), \mathbf{v}_{1}=(\rho, \mathbf{0})$. Очевидно, что $\left(t_{0}, \mathbf{v}_{0}\right) \in Q^{-}$и $\left(t_{1}, \mathbf{v}_{1}\right) \in Q^{+}$. Тогда согласно неравенству Гарнака имеем

$$
u\left(t_{0}, \mathbf{v}_{0}\right) \leqslant \max _{Q^{-}} u(t, x, \mathbf{y}) \leqslant \widehat{H} \min _{Q^{+}} u(t, x, \mathbf{y}) \leqslant \widehat{H} u\left(t_{1}, \mathbf{v}_{1}\right) .
$$

Далее, остается построить последовательность касающихся шаров $B\left(\rho_{i}, \mathbf{w}_{i}\right)$, первым и последним из которых являются соответственно шары $B(\rho ;(0, \mathbf{0}))$ и $B(\rho ;(1, \mathbf{0}))$, а затем записать неравенства, аналогичные $(5.2)$, взяв в качестве $\mathbf{v}_{i}$ точки касания шаров. При этом шары следует выбирать достаточно мальми, чтобы удвоенные шары лежали в $\widehat{M}$.

ЗАмЕчАниЕ. Легко видеть, что при гомотетиях и параллельных переносах области $\widehat{D}$ остается справедливьм неравенство вида (5.1).

ДоКАЗАТЕЛЬСТво ТЕОРЕМЫ 2 для простоты проведем для значения $b=4$.

Положим $\mathbf{v}_{j}=\left(x_{j}, \mathbf{0}\right), \rho_{j}=a\left(x_{j+1}-x_{j}\right) / 8, t_{0}=\rho_{0}^{2}, t_{j+1}=t_{j}+h\left(x_{j+1}-x_{j}\right)^{2}$, $j=\overline{0, \infty}$. Из условия (0.24) следуют неравенства

$$
\rho_{j+1} \leqslant 4 \rho_{j}, \quad j=\overline{0, \infty} .
$$

Индукцией по $j$ установим соотношения

$$
t_{j} \geqslant \rho_{j}^{2}, \quad j=\overline{0, \infty} .
$$


Действительно, по выбору $t_{0} \geqslant \rho_{0}^{2}$ и предположению индукции, пользуясь (5.3), получаем

$$
t_{j+1}=t_{j}+h\left(x_{j+1}-x_{j}\right)^{2} \geqslant \rho_{j}^{2}+16 \rho_{j}^{2} \geqslant \rho_{j+1}^{2} .
$$

Ввиду (5.4) и включений $\mathbf{v}_{j}+\left(x_{j+1}-x_{j}\right) \widehat{M} \subset \Omega$ по лемме 4 для пар $\left(t_{j}, \mathbf{v}_{j}\right)$, $\left(t_{j+1}, \mathbf{v}_{j+1}\right), j=\overline{0, \infty}$, устанавливаем неравенства

$$
u\left(t_{j}, \mathbf{v}_{j}\right) \leqslant H u\left(t_{j+1}, \mathbf{v}_{j+1}\right) .
$$

Конечно, при $j=0$ включение может не выполняться, но тем не менее точка $\left(x_{0}, \mathbf{0}\right)$ является внутренней для области $\Omega$, поэтому неравенство (5.5) будет также выполняться и при $j=0$, возможно с другой постоянной $H$.

Зафиксируем некоторое положительное число $t>t_{0}$ и положим $N=N(t)$. Пусть $r \leqslant N$ - первый номер, для которого $\delta(N+1)=\max _{j=\overline{0, N}} \Delta_{j}=x_{r+1}-x_{r}$. Далее, положим

$$
\rho_{j}=\rho_{r}, \quad t_{j}=t_{r}+\frac{a^{2}}{8} \delta(N+1)^{2}(j-r), \quad \mathbf{v}_{j}=\mathbf{v}_{r}, \quad j=\overline{r+1, \infty} .
$$

Очевидно, для пар $\left(t_{j}, \mathbf{v}_{j}\right),\left(t_{j+1}, \mathbf{v}_{j+1}\right), j=\overline{r, \infty}$, выполнено неравенство Гарнака.

Обозначим через $p$ первый номер, начиная с $p \geqslant r+1$, для которого $t_{p} \geqslant t$.

Согласно (5.1), ввиду того, что $\widehat{H} \leqslant H$, справедливо неравенство

$$
u\left(t_{p}, \mathbf{v}_{p}\right) \geqslant H^{-p} u\left(t_{0}, \mathbf{v}_{0}\right) .
$$

Более того, имеет место неравенство

$$
\min _{(x, \mathbf{y}) \in B\left(\rho_{r}, \mathbf{v}_{r}\right)} u\left(t_{p}, x, \mathbf{y}\right) \geqslant H^{-p} u\left(t_{0}, x_{0}, \mathbf{0}\right) .
$$

При этом справедливы соотношения

$$
p=r+(p-r) \leqslant N+\left(1+\frac{8 t}{a^{2} \delta^{2}(N+1)}\right) .
$$

Поскольку последовательность $\left\{x_{j}\right\}_{j=0}^{\infty}$ является $\lambda$-последовательностью, то справедливо неравенство (4.6). Применяя (4.6), полагая $N=N(t)$ и пользуясь определением (0.13) функции $N(t)$, выводим

$$
p \leqslant N+1+\frac{8 \theta t \lambda(N+1)}{a^{2} \epsilon}<\left(1+\frac{8 \theta}{a^{2} \epsilon}\right)(N(t)+1) .
$$

Учитьвая монотонное невозрастание функции $E(t) \equiv\|u(t)\|, t \geqslant 0$, заключаем справедливость следующих неравенств

$$
\begin{aligned}
& \int_{\Omega} u^{2}(t, x, \mathbf{y}) d x d \mathbf{y} \geqslant \int_{\Omega} u^{2}\left(t_{p}, x, \mathbf{y}\right) d x d \mathbf{y} \\
& \quad \geqslant \int_{B\left(\rho_{r}, \mathbf{v}_{r}\right)} u^{2}\left(t_{p}, x, \mathbf{y}\right) d x d \mathbf{y} \geqslant C_{1} \min _{(x, \mathbf{y}) \in B\left(\rho_{r}, \mathbf{v}_{r}\right)} u^{2}\left(t_{p}, x, \mathbf{y}\right) \rho_{r}^{n} .
\end{aligned}
$$


Используя (5.6), (5.7), при $t>t_{0}$ получаем

$$
\begin{aligned}
\|u(t)\|^{2} & \geqslant \frac{a^{n} C_{1}}{8^{n}} u^{2}\left(t_{0}, x_{0}, \mathbf{0}\right) \delta^{n}(N(t)+1) \exp \left[-(N(t)+1)\left(2+\frac{16 \theta}{a^{2} \epsilon}\right) \ln H\right] \\
& \geqslant C_{2} \delta^{n}(1) \exp \left[-C_{3} N(t)\right] .
\end{aligned}
$$

В условиях теоремы справедлива оценка (0.14) теоремы 1. В итоге для неотрицательного решения задачи при $t>T_{0}=\max \left(t_{0}, 2 / \lambda(2)\right)$ получаем неравенства (0.25). Теорема 2 доказана.

Построенная в $\S 4$ для области типа слоя $(0.15) \Pi^{l}$-последовательность $\left\{x_{j}\right\}_{j=0}^{\infty}$ при выполнении условия (0.21) удовлетворяет неравенству (4.18). Аналогичными рассуждениями доказывается неравенство

$$
\frac{\Delta_{j+1}}{\Delta_{j}} \geqslant \frac{1}{c^{\max (m / k, l / q)}}, \quad j=\overline{0, \infty}
$$

Поэтому имеет место условие (0.24). Ввиду вложений

$$
\left\{\left(x, y_{1}\right) \in \mathbb{R}_{2}: x_{j} \leqslant x \leqslant x_{j+1},-\Delta_{j}<y_{1}<\Delta_{j}\right\} \times \mathbb{R}_{n-1} \subset \Omega[f], \quad j=\overline{0, \infty},
$$

условие (0.23) при $k=q=l=m=1$ выполнено очевидньпм образом.

Автор выражает искреннюю благодарность $\Phi$. Х. Мукминову за предложенную тематику исследований, а также А.К. Гущину и В. П. Михайлову за стимулирующие обсуждения и полезные замечания.

\section{Список литературы}

1. Кондратьев В.А., Эйдельман С.Д. О свойствах решений линейных эволюционных систем с эллиптической пространственной частью // Матем. сб. 1970. Т. 81(123). C. $398-429$.

2. Гущин $A$. К. Об оценках решений краевых задач для параболического уравнения второго порядка // Труды МИАН. 1973. Т. 126. С. 5-45.

3. Гущин $A . K$. Стабилизация решений второй краевой задачи для параболического уравнения второго порядка // Матем. сб. 1976. Т. 101 (143). С. 459-499.

4. Уиаков В. И. Стабилизация решений третьей смешанной задачи для параболического уравнения в нецилиндрической области // Матем. сб. 1980. Т. 111(153). С. 95-115.

5. Лежнев A. В. О поведении при больших значениях времени неотрицательных решений второй смешанной задачи для параболического уравнения // Матем. сб. 1986. Т. 129. № 2. C. $186-200$.

6. Мукминов $\Phi . X$. Стабилизация решений первой смешанной задачи для системы уравнений Навье-Стокса // Дис. ... докт. физ.-матем. наук. М.: МИРАН, 1994.

7. Мукминов $\Phi . X$. Об убьвании нормы решения смешанной задачи для параболического уравнения высокого порядка // Диффференц. уравнения. 1987. Т. 23. № 10. С. 1172-1180.

8. Мукминов $\Phi . X$. Стабилизация решений первой смешанной задачи для параболического уравнения второго порядка // Матем. сб. 1980. Т. 111(153). № 4. С. 503-521.

9. Тедеев $A . \Phi$. Стабилизация решений первой смешанной задачи для квазилинейного параболического уравнения высокого порядка // Дифференц. уравнения. 1989. Т. 25. № 3. C. $491-498$. 
10. Биккулов И. М., Мукминов $\Phi . X$. О стабилизации нормы решения одной смешанной задачи для параболических уравнений 4-го и 6-го порядков в неограниченной области // Матем. сб. 2004. Т. 195. № 3. С. 115-142.

11. Кожевникова Л. М., Мукминов $\Phi$. Х. Оценки скорости стабилизации при $t \rightarrow \infty$ решения первой смешанной задачи для квазилинейной системы параболических уравнений второго порядка // Матем. сб. 2000. Т. 191. № 2. С. 91-131.

12. Хисамутдинова H. А. Стабилизация решения двумерной системы уравнений НавьеСтокса в неограниченной области с несколькими выходами на бесконечность // Матем. сб. 2003. Т. 194. № 3. С. 83-114.

13. Ладыженская О.А., Солонников В. А., Уральцева Н. Н. Линейные и квазилинейные уравнения параболического типа. М.: Наука, 1967.

14. Moser J. A. Harnack inequality for parabolic differential equations // Comm. Pure Appl. Math. 1964. V. 17. № 1. P. 101-134.

Стерлитамакская государственная

Поступила в редакцию

педагогическая академия

25.10 .2004

E-mail: kozul@mail.ru 